\title{
Graphene in inhomogeneous magnetic fields: Bound, quasi-bound, and scattering states
}

\author{
M. Ramezani Masir \\ Departement Fysica, Universiteit Antwerpen Groenenborgerlaan 171, B-2020 \\ Antwerpen, Belgium, \\ E-mail: mrmphys@gmail.com
}

\section{P. Vasilopoulos}

Department of Physics, Concordia University, Montreal, Quebec, Canada H3G 1M8.

E-mail: takis@alcor.concordia.ca

\section{F. M. Peeters}

Departement Fysica, Universiteit Antwerpen Groenenborgerlaan 171, B-2020

Antwerpen, Belgium.

E-mail: francois.peeters@ua.ac.be

\begin{abstract}
The electron states in graphene-based magnetic dot and magnetic ring structures and combinations of both are investigated. The corresponding spectra are studied as a function of the radii, the strengths of the inhomogeneous magnetic field and of a uniform background field, the strength of an electrostatic barrier, and the angular momentum quantum number. In the absence of an external magnetic field we have only long-lived quasi-bound and scattering states and we assess their influence on the density of states. In addition, we consider elastic electron scattering by a magnetic dot, whose average $B$ vanishes, and show that the Hall and longitudinal resistivities, as function of the Fermi energy, exhibit a pronounced oscillatory structure due to the presence of quasi-bound states. Depending on the dot parameters this oscillatory structure differs substantially for energies below and above the first Landau level.
\end{abstract}




\section{Introduction}

The study of graphene, a single layer of carbon atoms, has become a very active field of research in nanophysics [1]. It is expected that this material will serve as a basis for new electronic and opto-electronic devices. For that a control of the electron behaviour, using, e.g., electric fields, in this two-dimensional (2D) layer is necessary. This task though is made complicated by the Klein effect according to which Dirac electrons in graphene can tunnel, upon normal incidence, through any arbitrarily high and wide electric barrier [2]. Consequently, there are no bound states in electrically created quantum dots but only quasi-bound states[3, 4, 5], which, however, can have a long lifetime under certain conditions.

An alternative approach to control the motion of electrons is to use inhomogeneous magnetic fields which can be created, e.g., by the deposition of nanostructured ferromagnets $[6,7]$. The resulting inhomogeneous magnetic structures, mainly magnetic barriers or steps, are able to confine Dirac electrons in graphene $[8,9,10,11,12,13,14]$. In particular, the spectra of simple dots and rings were studied in Refs. [8, 15, 16] and electron scattering by a magnetic ring in Ref. [17]. With the exception of Ref. [10] electrostatic barriers were not included in these treatments. However, these inhomogeneous structures are relatively simple and a detailed account of the resulting bound or quasi-bound states for more complex profiles is, to our knowledge, missing. Furthermore, most of the results were discussed as a function of the strength of the inhomogeneous magnetic field which is not easy to vary experimentally. Here we investigate the effect of an external homogenous magnetic field, which can be tuned experimentally, on the electron states.

In more detail, we consider finite-size magnetic structures in which the magnetic field is inhomogeneous and treat bound, quasi-bound, and scattering states with emphasis on the latter. We do that for a magnetic dot and a ring in the presence or absence of a tunable external homogeneous magnetic field. In addition, we study a specific profile in which a dot-shaped magnetic field profile with field $-B$, is surrounded by a ring-shaped magnetic field profile, of finite width, with field $B$ and evaluate the quasi-bound states. Such inhomogeneous magnetic field profiles can be realized by having a magnetic vortex piercing the graphene layer or by covering graphene with a type I superconductor with a circular hole or annulus placed in a perpendicular magnetic field. Another way is by deforming the lattice locally; this results in a local strain which in graphene can induce an effective inhomogeneous magnetic field [18, 19]. We also include in the calculation electrostatic potential barriers.

In previous work it was shown that it is impossible to confine $2 \mathrm{D}$ electrons in a magnetic dot, in contrast to semi-infinite magnetic structures, since all Landau levels (LLs) convert themselves into unbound states. Nevertheless, long-living, quasi-bound states can be present [20]. In view of that we carry out a similar study but for more complex magnetic field profiles, in the presence or absence of an external magnetic field, and show that in its presence bound states do exist. We focus on the behavior of 
the quasi-bound states and show that they affect various properties, e.g., the density of states and the longitudinal and Hall resistivities. In particular, we consider electron scattering by a magnetic dot with zero average $B$ and evaluate the Hall and longitudinal resistivities as a function of the electron energy.

The paper is organized as follows. In Sec. 2 we present the basic formalism and discuss briefly the results for a homogeneous magnetic field profile. In Sec. 3 we apply this formalism to the inhomogeneous magnetic field profiles of a magnetic dot and a magnetic ring, as well as to more complex profiles in Sec. 4, and focus on the spectra and the local density of states. We treat in some detail electron scattering by a magnetic dot, in which the average field $B$ vanishes, in Sec. 5 and show that the Hall and longitudinal resistivities oscillate with the Fermi energy. Our conclusions follow in Sec. 6.

\section{Basic formalism}

We consider electrons in graphene in a perpendicular magnetic field $B$. The relevant eigenvalue problem is

$$
\{H-E\} \Psi(r)=0 \text {. }
$$

The Hamiltonian of a massless Dirac electron, with momentum $\mathbf{p}$, near the $\mathbf{K}$ point is given by

$$
H=v_{F} \sigma \cdot(\mathbf{p}+e \mathbf{A})=\left(\begin{array}{cc}
0 & -i p_{+}+A_{-} \\
-i p_{+}+A_{+} & 0
\end{array}\right)
$$

where $p_{ \pm}=\partial / \partial x \pm i \partial / \partial y, \sigma=\left(\sigma_{x}, \sigma_{y}\right)$ the Pauli matrices and $A_{ \pm}=A_{x} \pm i A_{y}$, $\mathbf{A}=\left(A_{x}, A_{y}\right)$ the vector potential and $v_{F}$ the Fermi velocity. We obtain the Hamiltonian near the $\mathbf{K}^{\prime}$ point by replacing $\sigma$ with $\sigma^{*}$ in Eq. (2). From now on we restrict ourselves to electron states near the $\mathbf{K}$ point because they are decoupled from those of an electron near the $\mathbf{K}^{\prime}$ point of the Brillouin zone. To simplify the notation we introduce the dimensionless variables $B \rightarrow B_{0} B, A \rightarrow B_{0} R A, t \rightarrow t R / v_{F}, \vec{r} \rightarrow R \vec{r}, \vec{v} \rightarrow v_{F} \vec{v}, E \rightarrow$ $E_{0} E, E_{0}=\hbar v_{F} / R, \gamma_{i}=e B_{i} R^{2} / 2 \hbar$. $R$ is a typical length scale of our problem taken e. g. equal to the radius of the dot.

In view of the circular symmetry of the magnetic and potential profiles we will use polar coordinates. This is accomplished by the relations $x \pm i y=r e^{ \pm i \varphi}$ and $p_{ \pm}=e^{ \pm i \varphi}[\partial / \partial r \pm(i / r) \partial / \partial \varphi]$. We look for solutions that are periodic in the angle $\varphi$. We can write the two-component wave function $\Psi$ in the form

$$
\Psi=\left(\begin{array}{c}
\psi_{1}(r, \varphi) \\
\psi_{2}(r, \varphi)
\end{array}\right)=e^{i m \varphi}\left(\begin{array}{c}
a(r) \\
i e^{i \varphi} b(r)
\end{array}\right)
$$

with $m=\cdots,-2,-1,0,1,2, \cdots$. Combining Eqs. (1) and (3) we find that the components $a(r)$ and $b(r)$ must satisfy the following coupled first-order differential equations

$$
\begin{aligned}
& \{d / d r+g(r)+(m+1) / r\} b(r)=E a(r), \\
& -\{d / d r-g(r)-m / r\} a(r)=E b(r),
\end{aligned}
$$


with $g(r)=\gamma_{i} r+s_{i} / r$ where the coefficients $\gamma_{i}$ and $s_{i}$ are determined by the specific magnetic field profile. It is convenient to transform Eqs. (4) and (5) to a single, secondorder equation. We readily find that the equation for the component $b(r)$ reads

$$
\left\{\frac{1}{r} \frac{d}{d r} r \frac{d}{d r}+\left[-\gamma_{i}^{2} r^{2}-P_{i}^{2} / r^{2}+Q_{i}^{2}\right]\right\} b(r)=0,
$$

with $P_{i}^{2}=\left(s_{i}+m+1\right)^{2}$ and $Q_{i}^{2}=E^{2}-2 \gamma_{i}\left[s_{i}-(m+1)\right]$. A similar equation is obtained for the component $a(r)$. The solution of Eq. (6) is

$$
b(r)=r^{\left|P_{i}\right|} e^{-\left|\gamma_{i}\right| r^{2} / 2}\left\{A_{i} M\left(\alpha_{i}, \beta_{i}, z_{i}\right)+B_{i} U\left(\alpha_{i}, \beta_{i}, z_{i}\right)\right\},
$$

with $\alpha_{i}=\left(\left|P_{i}\right|+1\right) / 2-Q_{i}^{2} / 4\left|\gamma_{i}\right|, \beta_{i}=\left|P_{i}\right|+1$, and $z_{i}=\left|\gamma_{i}\right| r^{2}$. Here $M(\ldots)$ and $U(\ldots)$ are the confluent hypergeometric functions [21]. From Eq. (4) we obtain

$$
a(r)=\frac{1}{E}\left[2\left|\gamma_{i}\right| r \frac{d}{d z_{i}}+\left(\left(\gamma_{i}-\left|\gamma_{i}\right|\right) r+2\left|P_{i}\right| / r\right)\right] b(r) .
$$

The solution for the component $a(r)$ in a homogenous field $B$, with the unit of length $R$ taken equal to the magnetic length $\ell_{B}=(\hbar / e B)^{1 / 2}$ (i. e. $\gamma_{i}=1 / 2, s_{i}=0$ and $\left.E_{0}=\hbar v_{F} / \ell_{B}\right)$, can be written as

$$
a_{-}(r)=A r^{|m|} e^{-r^{2} / 4} M\left(\left(|m|+m+2-E^{2}\right) / 2,|m|+1, r^{2} / 2\right) .
$$

The above solution is finite in all regions provided the first argument of the function $M(\ldots)$ is a negative integer or zero, namely, $\left(|m|+m+2-E^{2}\right) / 2=-n, n=0,1,2, \cdots$. This gives the energy $E \equiv E_{n m}$

$$
E_{n m}=\sqrt{2 n+|m|+m+2} .
$$

Thus, we have the standard expression for the LLs of a Dirac electron starting with the level

$$
E_{00}=\sqrt{2} .
$$

This is not the lowest LL. Thus, let us consider the equation for the component $b(r)$. Its solution reads

$$
\begin{aligned}
& b(r)=A r^{|m+1|} e^{-r^{2} / 4} \\
& \times M\left(\left(|m+1|+m+1-E^{2}\right) / 2,|m+1|+1, r^{2} / 2\right) .
\end{aligned}
$$

Setting the first argument of $M$ equal to a negative integer $-n$ gives

$$
E_{n m}=\sqrt{2 n+|m+1|+m+1} .
$$

This expression for the LLs coincides with that given by Eq. (10) except for the lowest level which is

$$
E_{0,-1}=0
$$

These chiral levels are topologically protected by the Atyah-Singer index theorem[22]. From the above derivations it follows that zero energy states reside only in one of the two sublattices of graphene which are determined by the sign of the magnetic field. One consequence of that is that the lowest LL for Dirac electrons has half the degeneracy 
of the other LLs whether the problem is solved in the Landau gauge [23] or in the symmetric gauge. It also holds for the original Dirac equation, involving the real spin of an electron and not the pseudospin as in graphene, in either gauge [24]. In particular, the number of zero energy states is independent of the shape of the magnetic field profile and is proportional to the total magnetic flux $[22,25,26]$.

(a)

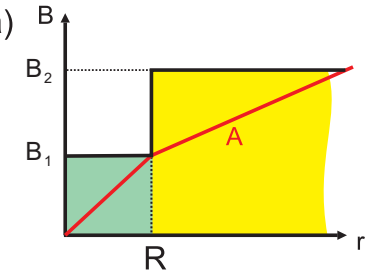

(b)

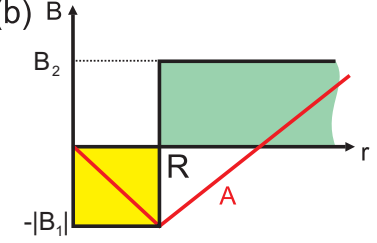

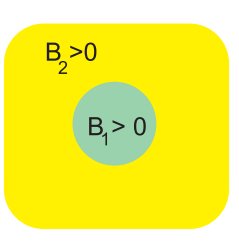

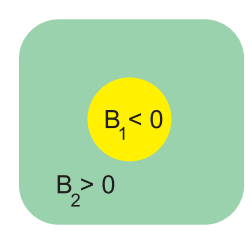

Figure 1. Two configurations of a magnetic dot, created by a field $B_{1}$, in an external magnetic field $B_{2}$. The corresponding magnetic field (black lines) and vector potential (red lines) profiles are shown in the right panels.

\section{Bound states}

\subsection{Magnetic dot}

Quasi-bound states in a magnetic dot were treated before in Ref. [20]. Here we focus on the bound states that occur when the dot is placed in a uniform magnetic field; in doing so we generalize the results of Ref. [8] valid for zero background field. The situation is depicted in Fig. 1 and the corresponding vector potential is given by

$$
A(r)=\left\{\begin{array}{ll}
\gamma_{1} r ; & 0<r<1, \\
\gamma_{2} r+\left(\gamma_{1}-\gamma_{2}\right) / r ; & r>1,
\end{array},\right.
$$

where $r$ is the radial coordinate in units of $R$, the dot radius. Then the solutions for $b(r)$ in the two regions, inside the dot $r<1$ and outside it $r>1$, are

$$
b(r)=\left\{\begin{array}{l}
A r^{\left|P_{1}\right|} e^{-\left|\gamma_{1}\right| r^{2} / 2} M\left(\alpha_{1}, \beta_{1}, z_{1}\right) ; \quad 0<r<1, \\
B r^{\left|P_{2}\right|} e^{-\left|\gamma_{2}\right| r^{2} / 2} U\left(\alpha_{2}, \beta_{2}, z_{2}\right) ; \quad r>1 .
\end{array}\right.
$$

Those for the component $a(r)$ can be found from Eq. (4).

To investigate possible bound states we match the wave functions at $r=1$ using $a^{-}(1)=b^{-}(1), a^{+}(1)=b^{+}(1)$. This leads to the nonlinear equation

$$
\left[b^{+}(r) a^{-}(r)-b^{-}(r) a^{+}(r)\right]_{r=1}=0,
$$

where $+(-)$ refers to region inside (outside) the dot. The solution of this nonlinear 


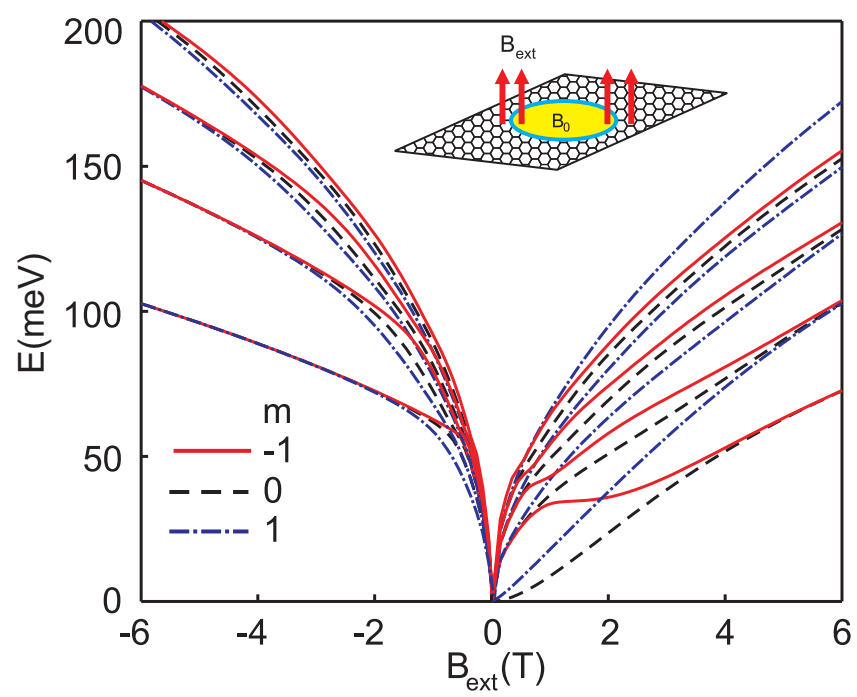

Figure 2. (a) Energy spectrum versus external magnetic field $B_{\text {ext }}$ for the circular magnetic field profile shown in the inset with $B_{0}=-2 T, R=50 \mathrm{~nm}$, and $-1 \leq m \leq 1$.

equation gives us the energy spectrum of the bound states. The numerical results as a function of the background magnetic field $B_{\text {ext }}$ are shown in Fig. 2 for three different values of the angular momentum $m$. Notice that: i) for $\left|B_{\text {ext }}\right| \gg\left|B_{0}\right|$ the usual LLs of electrons in graphene are recovered, and ii) for $B_{\text {ext }} \rightarrow 0$ there are no bound states as discussed previously in Ref. [20]. In Fig. 3(a) the energy states are plotted as a function of the dot radius with the magnetic field inside the dot, chosen as $B_{1}=-B_{2} / 2$, and the ratio of the corresponding LLs inside and outside the dot being $E_{\text {out }}^{L L}=\sqrt{2} E_{\text {in }}^{L L}$. This means that for quantum numbers $n_{\text {out }}=2 n_{\text {in }}$ the LLs inside and outside the dot have the same values. Since the magnetic fields inside and outside the dot have opposite sign, we have bonding and anti-bonding states [29] as is clearly apparent for $n=2$ in Fig. $3(\mathrm{a})$.

As is clear from Fig. 3(a), for $R$ small the electron is mainly outside the dot with an energy corresponding to a LL determined by the magnetic field outside the dot. When $R$ increases the bonding state will decrease in energy because it samples a smaller average magnetic field. The electron state is partially inside and outside the dot. Only when $R$ is large enough will the electron be localized inside the dot. We show the total wave function, corresponding to the states marked by open dots in Fig. 3(a), for $m=0, \pm 1$ in Fig. 3(b). For these values of $m$ the electron is on average located around the boundary at $r=R=30 \mathrm{~nm}$.

Using $j_{x, y}=v_{F} \Psi^{\dagger} \sigma_{x, y} \Psi$, the total angular current is

$$
J_{\varphi}=v_{F} \Psi^{\dagger}\left(\sigma_{y} \cos \varphi-\sigma_{x} \sin \varphi\right) \Psi=v_{F} \Psi^{\dagger} \zeta_{\varphi} \sigma_{y} \Psi
$$

with $\zeta_{\varphi}$ given by

$$
\zeta_{\varphi}=\left(\begin{array}{cc}
\mathrm{e}^{-\mathrm{i} \varphi} & 0 \\
0 & \mathrm{e}^{\mathrm{i} \varphi}
\end{array}\right) .
$$



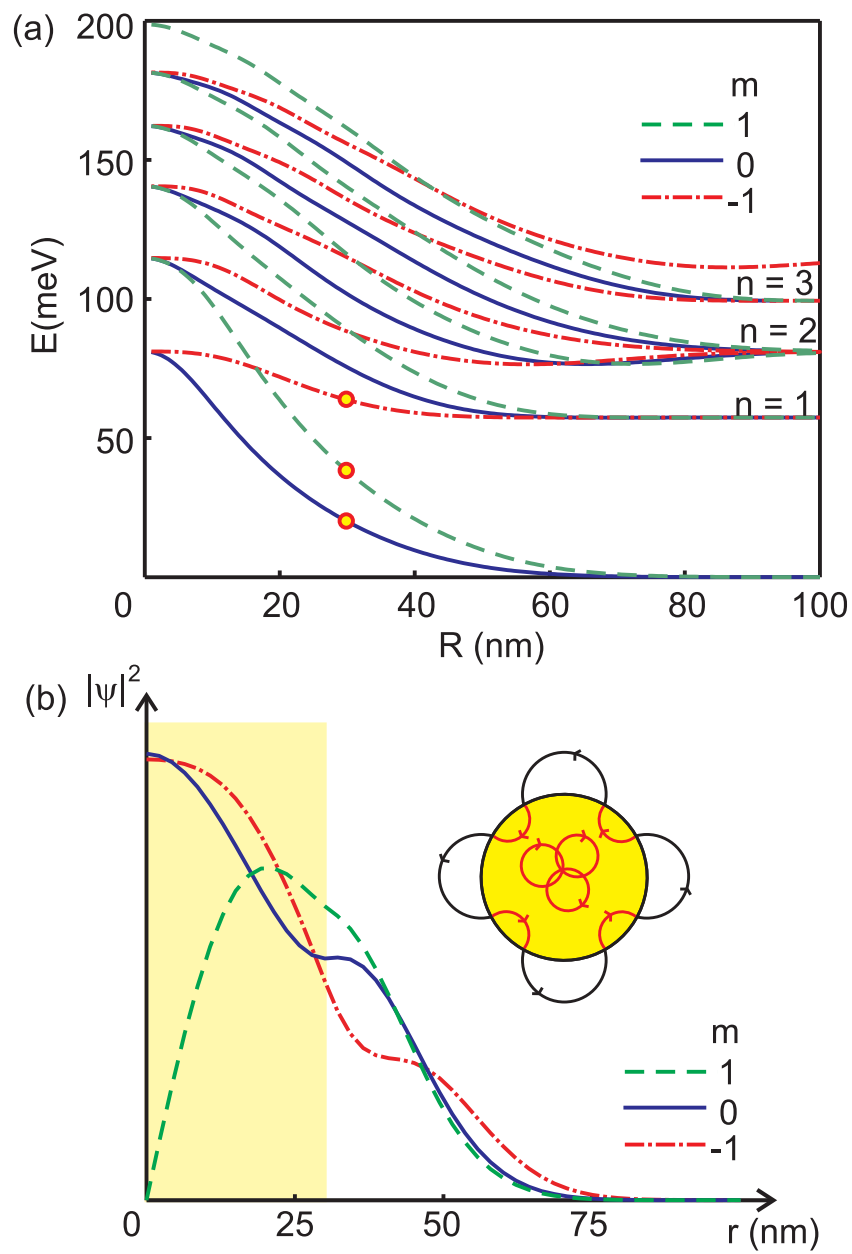

Figure 3. (a) Energy spectrum versus dot radius for the magnetic field profile shown in Fig. 1 with $B_{1}=-2.5 T, B_{2}=5 \mathrm{~T}$, and $-1 \leq m \leq 1$. (b) The amplitude squared of the wave function of the eigenstates, marked by open red dots in panel (a), versus the radial coordinate for $R=30 \mathrm{~nm}$. The inset shows a classical snake trajectory.

For the special case $B_{2}=-B_{1}=B$ we show the two split levels, for $m=0$ and 1 in Fig. 4 (a) and the angular current $J_{\varphi}$ in Fig. 4 (b) for $R=40 \mathrm{~nm}$. Notice that at the edge $r=R=40 \mathrm{~nm}, J_{\varphi}$ is negative (positive) for bonding (anti-bonding) states. The insets in Fig. 4(b) show schematically the corresponding semi-classical electron trajectories.

In Fig. 5(a) we plot the energy states as a function of the radius for a dot with positive magnetic field inside and outside the dot. In this case the classical trajectories inside and outside the dot rotate in the same direction, see inset to Fig. 5 (b); consequently, there are no anti-bonding states but only bonding states. The wave function amplitudes, for three different states, are plotted in Fig. 5 (b) for $R=30 \mathrm{~nm}$ and they are rather similar with those shown in Fig. 3(b).

In Fig. 6 the energy states are plotted as a function of the quantum number $m$ for $B_{1}=-B_{2}$ and $R=50 \mathrm{~nm}$. As shown in Fig. 6(a), with increasing $m$ all levels split into bonding and anti-bonding states. This is not so when $B_{1}=2.5 \mathrm{~T}$ and $B_{2}=5 \mathrm{~T}$ as 

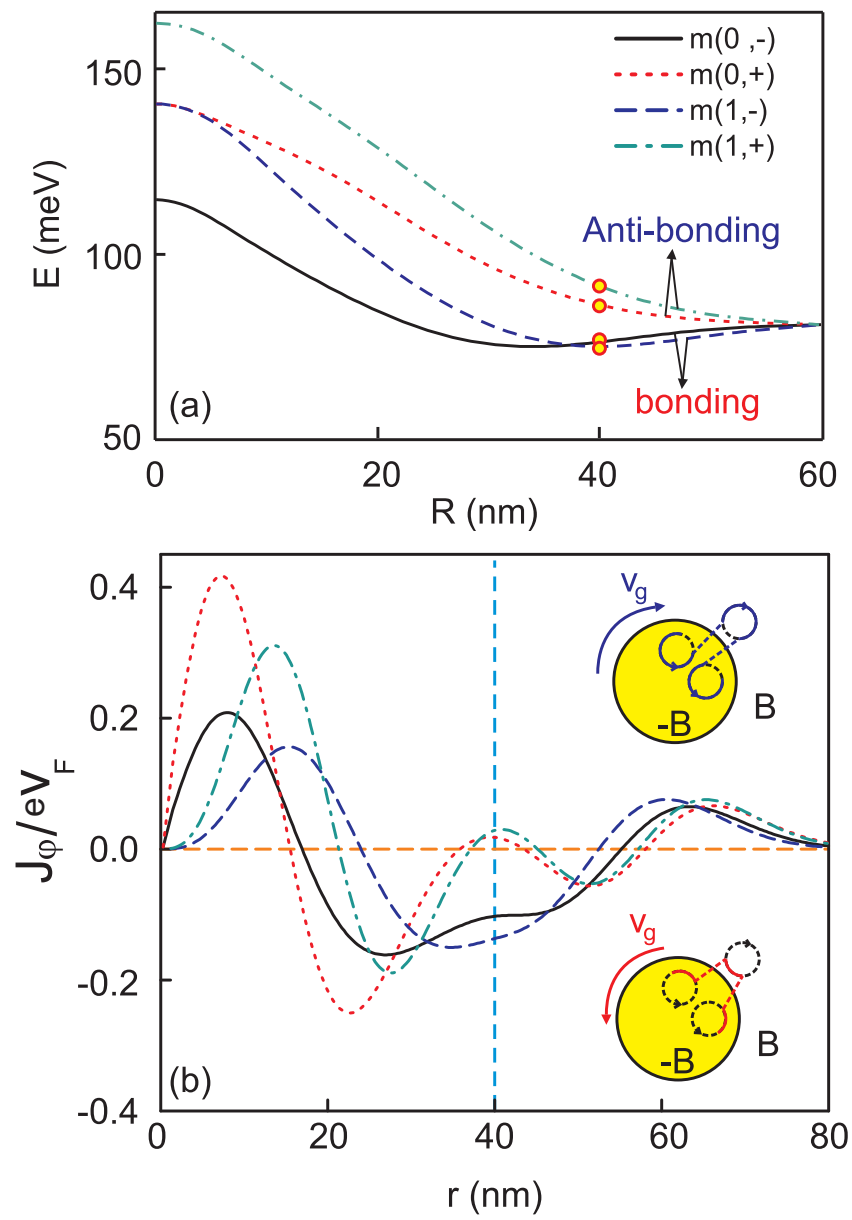

Figure 4. (a) Energy spectrum for the magnetic field profile shown in Fig. 1 with $B_{1}=-5 T, B_{2}=5 \mathrm{~T}, n=1$, and $m=0,1$. The - and + signs denote bonding and anti-bonding states, respectively. (b) Angular current versus $r$ for the eigenstates marked by open, red dots in panel (a). The lower (upper) inset shows a schematic semi-classical interpretation of the bonding (anti-bonding) states.

shown in Fig. 6(b).

In the presence of a constant 2D potential $V(x, y)=V \Theta(R-r)$ one has to add this term in Eq. (2). The analytical form of the wave functions remain the same in all regions of interest but the eigenvalues do not. Accordingly, the degeneracies shown in Figs. 3(a) and 4(a) should be lifted. Indeed, this is the case as we show in Fig. 7 where we plot the spectrum as a function of the dot radius for antiparallel fields in (a) $\left(B_{1}=-5 T, B_{2}=5 \mathrm{~T}\right)$, and parallel fields in (b) $\left(B_{1}=2.5 \mathrm{~T}, B_{2}=5 \mathrm{~T}\right)$ in the presence of the constant potentials $V_{1}=20 \mathrm{meV}$ inside the dot and $V_{2}=0$ outside the dot. The curves shown correspond to $m=0, \pm 1, \pm 2$.

We now investigate whether a Dirac electron can have a zero energy state inside the dot. To find such a state we set $E=0$ in Eqs. 4(a) and 4(b). Then the general solutions are

$$
a(r) \sim r^{m+s_{i}} \times e^{\gamma_{i} r^{2} / 2}
$$


Graphene in inhomogeneous magnetic fields: Bound, quasi-bound, and scattering states9
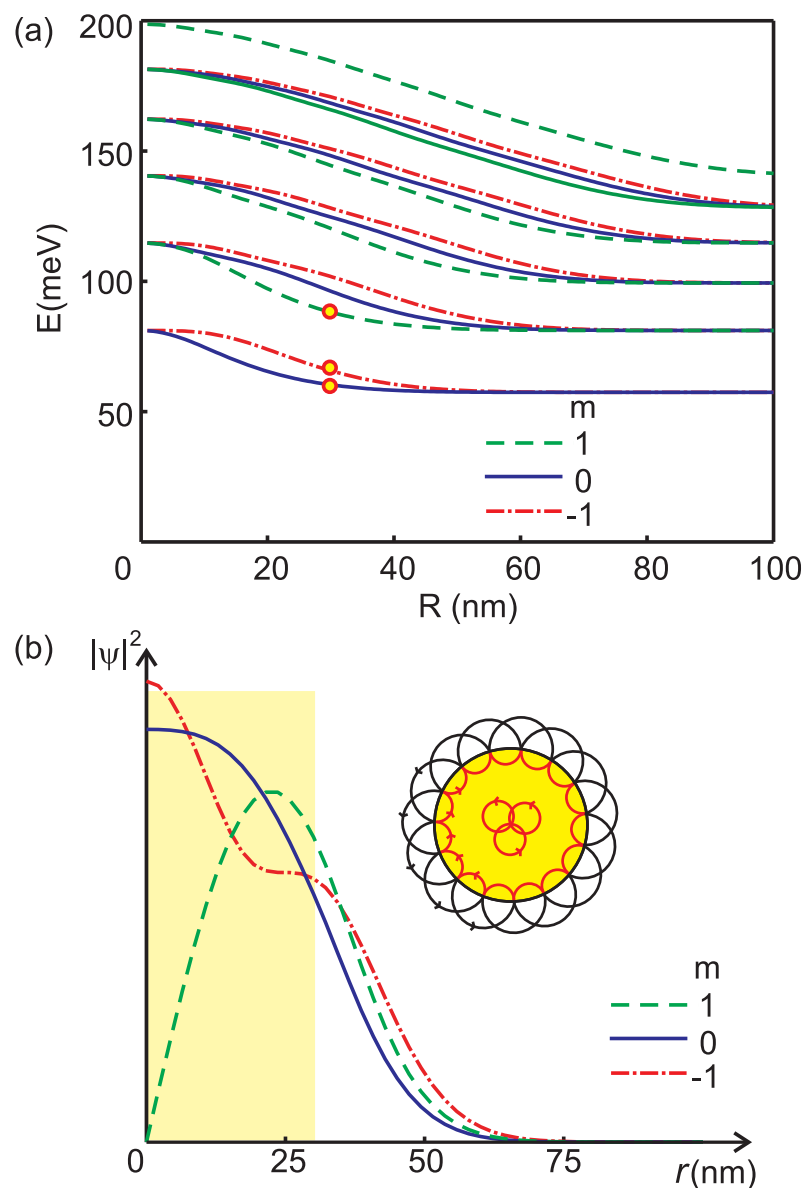

Figure 5. (a) Energy spectrum for the magnetic field profile shown in Fig. 1 with $B_{1}=2.5 \mathrm{~T}, B_{2}=5 \mathrm{~T}$, and $-1 \leq m \leq 1$. (b) The wave function for the eigenstates marked by a dot in panel (a). The inset shows the classical trajectories inside and outside the dot rotating in the same direction
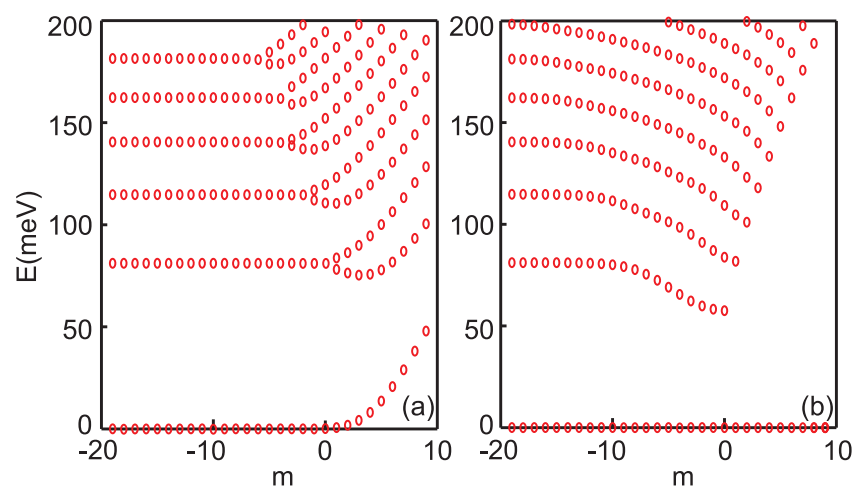

Figure 6. (a) Dependence of the energy spectrum on the quantum number $m$ in a magnetic dot with $R=50 \mathrm{~nm}$ and for (a) $B_{1}=-5 \mathrm{~T}$ and $B_{2}=5 \mathrm{~T}$ and (b) $B_{1}=2.5$ $\mathrm{T}$ and $B_{2}=5 \mathrm{~T}$. 
Graphene in inhomogeneous magnetic fields: Bound, quasi-bound, and scattering states10
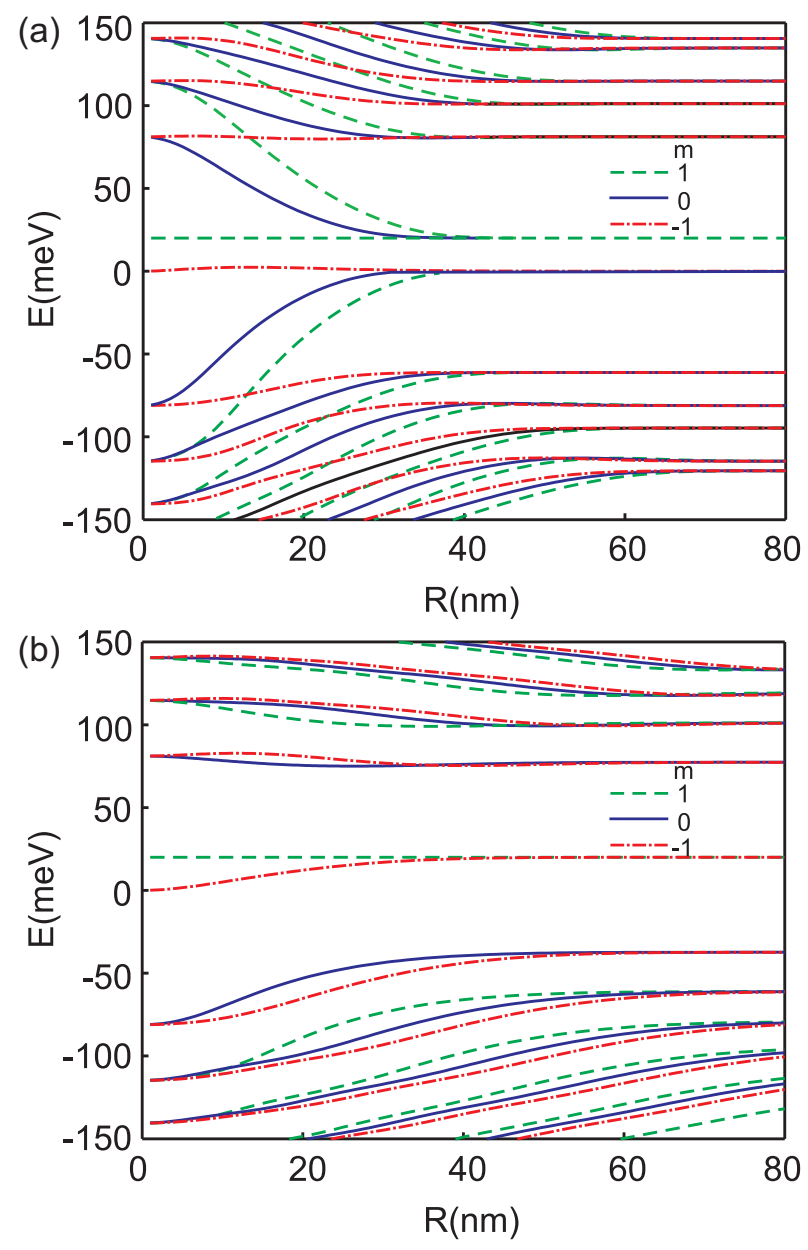

Figure 7. (a) Energy spectrum for the magnetic field profile shown in Fig. 1 with $B_{1}=-5 T, B_{2}=5 \mathrm{~T}$, and $-2 \leq m \leq 2$, in the presence of a potential step formed by $V_{1}=20 \mathrm{meV}$ and $V_{2}=0$. (b) The same as in (a) with $B_{1}=2.5 T$.

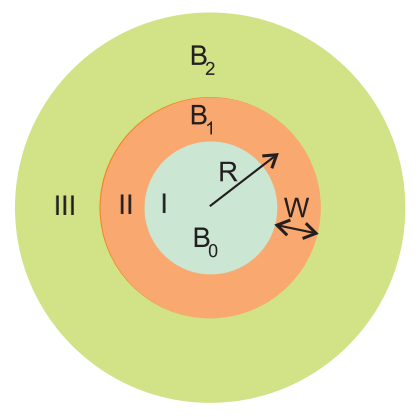

Figure 8. An inhomogeneous magnetic field profile involving a ring of width $W$. As indicated, the field has the values $B_{0}$ inside the hole of the ring, $B_{1}$ in the ring area, and $B_{2}$ outside the ring.

and

$$
b(r) \sim r^{-m-1-s_{i}} \times e^{-\gamma_{i} r^{2} / 2} .
$$


Graphene in inhomogeneous magnetic fields: Bound, quasi-bound, and scattering states11

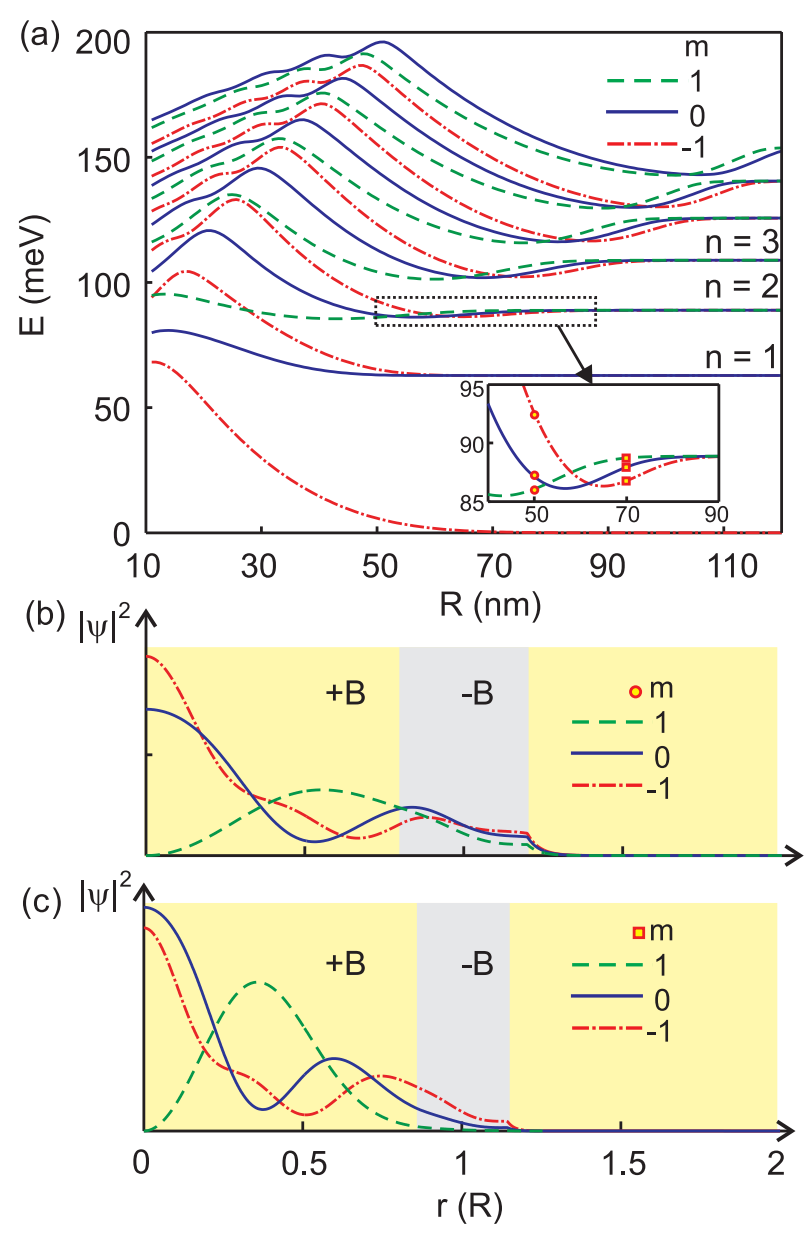

Figure 9. (a) Energy spectrum as a function of $R$ for the ring shown in Fig. 8 with $B_{0}=B_{2}=3 \mathrm{~T}, B_{1}=-3 \mathrm{~T}$ for $m=0, \pm 1$, and $W=20 \mathrm{~nm}$. (b) Wave function for the eigenstates marked by dots or squares in the inset of panel (a)

In order to have a finite wave function we have to satisfy the boundary conditions. First, at $r=0$ the wave function should behave as $r^{\alpha}(\alpha \geq 0)$ and for $r \rightarrow \infty$ the function should decay like $r^{-\alpha}(\alpha \geq 1)$. These conditions for the component $a$ entail $m \geq-s_{1}$ if $r \rightarrow 0$ and $\gamma_{2}<0$ if $r \rightarrow \infty$. For the component $b(r)$, these conditions lead to $m \leq-s_{1}-1$ for $r \rightarrow 0$ and to $\gamma_{2}>0$ for $r \rightarrow \infty$.

For a magnetic dot in a homogeneous background magnetic field, the field inside and outside the dot is different. For example, for $\gamma_{1}<0$ and $\gamma_{2}>0$ we have $s_{1}=0$ and $s_{2}=\gamma_{1}-\gamma_{2}$. In order to satisfy these conditions $a(r)$ should be zero, otherwise a divergency occurs at $r \rightarrow \infty$.

For the second component $b$ the condition reads, $m \leq-1$. Then the number of degenerate, zero-energy states is of order $\approx L^{2}$, where $L$ is the size of the sample. Note that the number of zero-energy states relates to the total magnetic flux which here diverges because the area of the magnetic dot, as compared to the sample area, is too small, i. e. $L \gg R$, and the magnetic flux is proportional to $\approx L^{2}$. 
Graphene in inhomogeneous magnetic fields: Bound, quasi-bound, and scattering states12
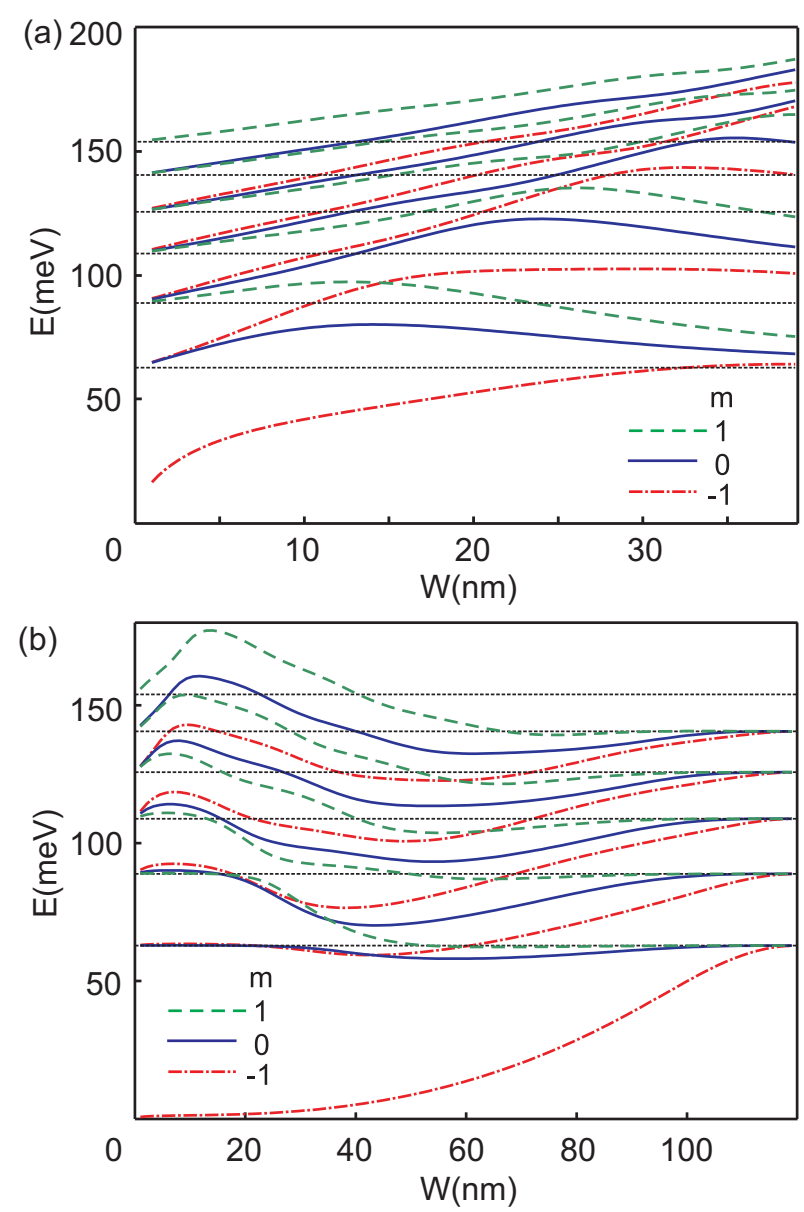

Figure 10. (a) Energy spectrum as a function of $W$ for the ring shown in Fig. 8 with $B_{0}=B_{2}=3 \mathrm{~T}, B_{1}=-3 \mathrm{~T}$ for $-1 \leq m \leq 1$, (a) for $R=20 \mathrm{~nm}$ and (b) $R=50 \mathrm{~nm}$. The thin dashed horizontal black lines show the position of the LLs in a homogenous magnetic field $B=3$ T.

\subsection{Magnetic ring}

Now we look for possible bound states in the magnetic ring shown in Fig. 8. With $R_{1}=R-W / 2$ and $R_{2}=R+W / 2$ the vector potential for this magnetic field profile is

$$
A(r)= \begin{cases}\gamma_{1} r, & 0<r<R_{1}, \\ \gamma_{2} r+\frac{\left(\gamma_{1}-\gamma_{2}\right) R_{1}^{2}}{r}, & R_{1}<r<R_{2}, \\ \gamma_{3} r+\frac{\left(\gamma_{2}-\gamma_{3}\right) R_{2}^{2}+\left(\gamma_{1}-\gamma_{2}\right) R_{1}^{2}}{r}, & r>R_{2} .\end{cases}
$$

The solutions for $b(r)$ in the three different regions are

$$
b(r)=\left\{\begin{array}{lc}
A r^{\left|P_{1}\right|} e^{-\left|\gamma_{1}\right| r^{2} / 2} M\left(\alpha_{1}, \beta_{1}, z_{1}\right), & 0<r<R_{1}, \\
r^{\left|P_{2}\right|} e^{-\left|\gamma_{2}\right| r^{2} / 2}\left[C M\left(\alpha_{2}, \beta_{2}, z_{2}\right)+D U\left(\alpha_{2}, \beta_{2}, z_{2}\right)\right], & R_{1}<r<R_{2}, \\
B r^{\left|P_{3}\right|} e^{-\left|\gamma_{3}\right| r^{2} / 2} U\left(\alpha_{3}, \beta_{3}, z_{3}\right), & r>R_{2} .
\end{array}\right.
$$


Graphene in inhomogeneous magnetic fields: Bound, quasi-bound, and scattering states13

(a)

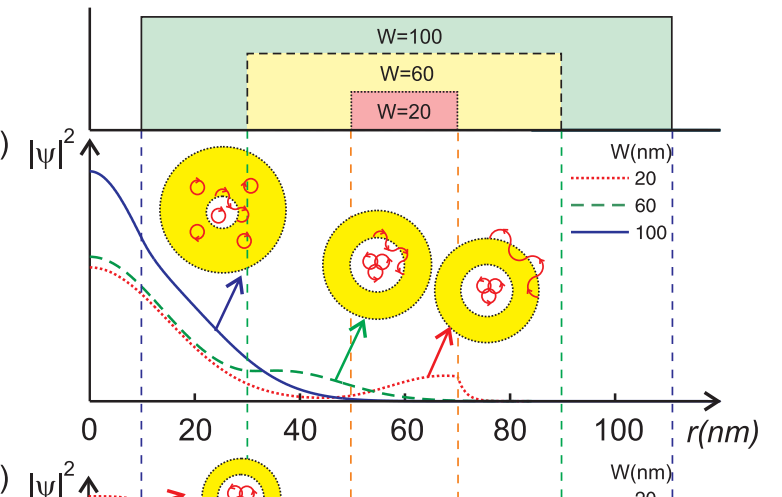

(b)

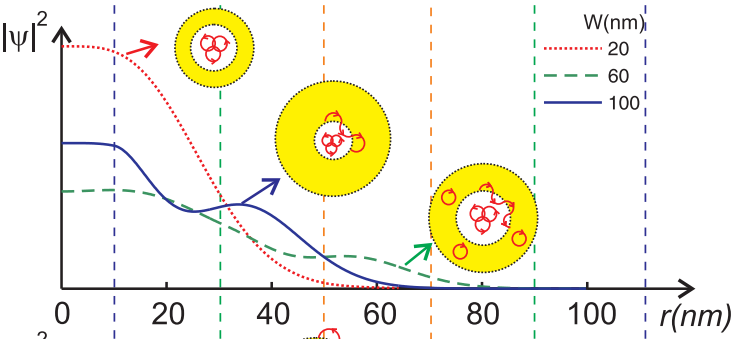

(c)

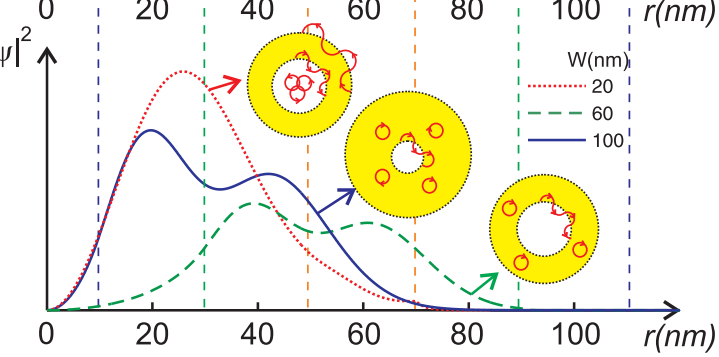

Figure 11. (a) The total wave functions for the lowest eigenstates with $m=-1$, $W=20,60,100 \mathrm{~nm}$ and $R=60 \mathrm{~nm}$. (b) and (c) as in (a) for $m=0$ and $m=1$, respectively. The insets show the classical trajectories.

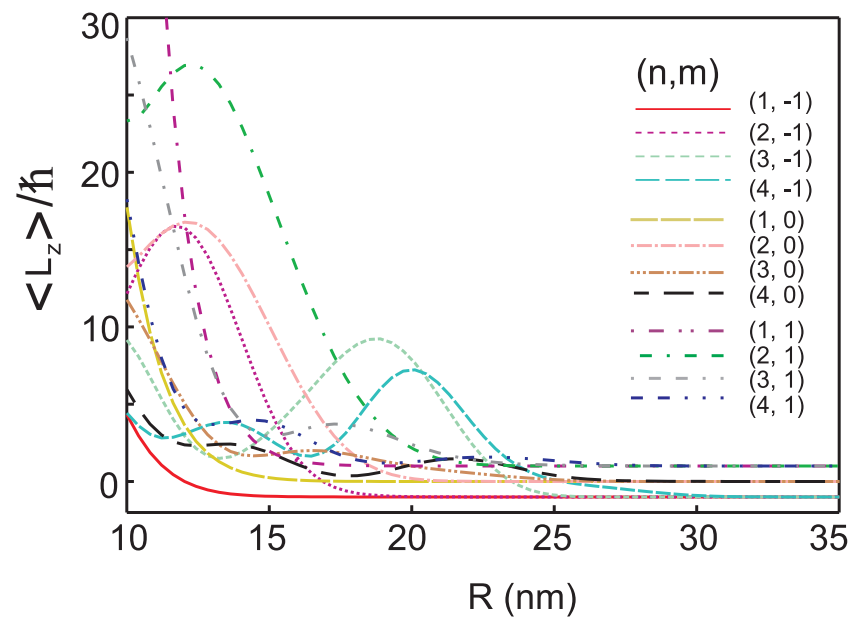

Figure 12. Expectation value of the angular momentum $L_{z} / \hbar$ as function of $R$ for the ring shown in Fig. 8 with $B_{0}=B_{2}=3 \mathrm{~T}, B_{1}=-3 \mathrm{~T},-1 \leq m \leq 1$, and $W=20$ $\mathrm{nm}$. 

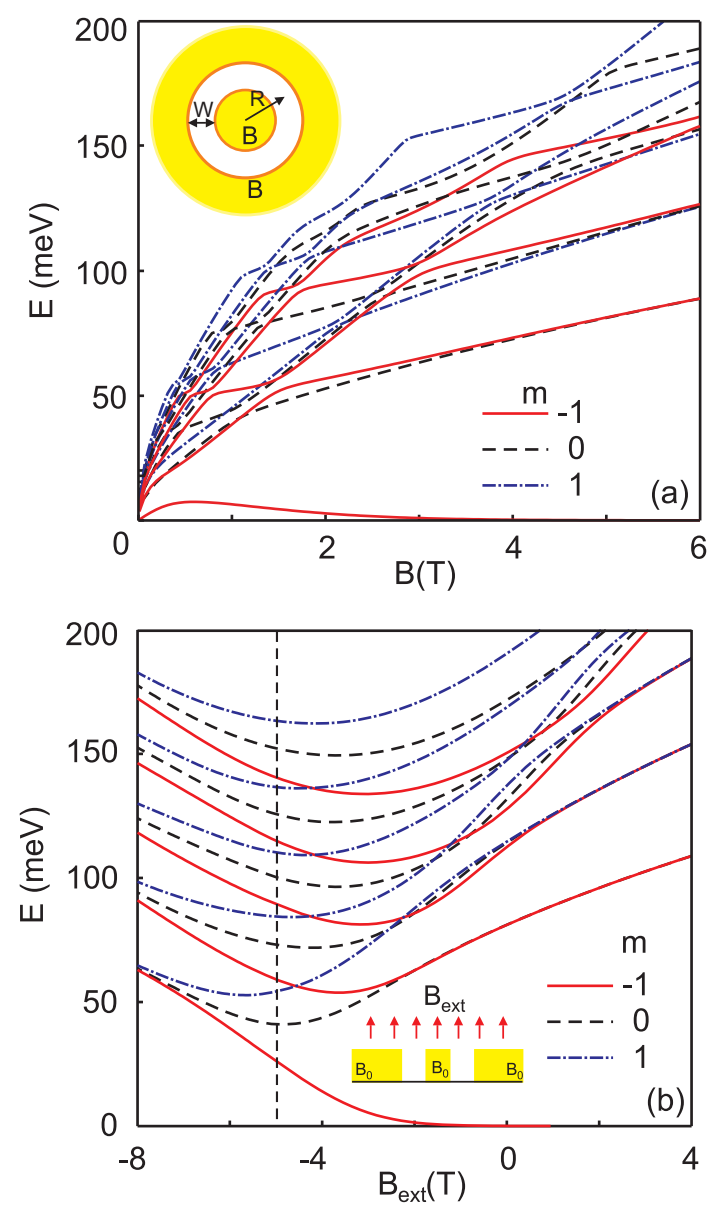

Figure 13. (a) Energy spectrum as a function of the magnetic field $B$ for the ring shown in the inset. (b) Energy spectrum for a magnetic ring as a function of an external magnetic field with $B_{0}=5 \mathrm{~T}$ (see inset). The vertical dashed line indicates where $B_{0}=-B_{\text {ext }}$. In both cases $R=60 \mathrm{~nm}$ and $W=20 \mathrm{~nm}$.

with $\alpha_{i}, \beta_{i}$, and $z_{i}$ as given just below Eq. (6).

The component $a(r)$ is obtained from Eq. (4). We have to satisfy the boundary conditions at $R_{1}=R-W / 2$ and $R_{2}=R+W / 2$ which then results in a non-linear equation for the energy spectrum. In Fig. $9(\mathrm{a})$ we plot the bound states obtained for a ring with $B_{1}$ antiparallel to $B_{0}$ and $B_{2}$ and all of them equal to $3 \mathrm{~T}$. The width of the ring $(W=20 \mathrm{~nm})$ is fixed and we plot the bound states as a function of $R$. With increasing $R$ all bound states approach the LL energies for a homogenous field $B=3 \mathrm{~T}$. The inset shows how the order of the levels is reversed between $R=50 \mathrm{~nm}$ and $R=70$ nm.

We plot the amplitude of the wave functions in Figs. 9(b) and 9(c) for the states marked by circle and squares at $R=50 \mathrm{~nm}$ and $R=70 \mathrm{~nm}$, respectively. Note that when $R=50 \mathrm{~nm}$ the electron is mainly inside the hole of the ring, but there is a finite probability to find the electron near the boundaries $R=R_{1}$ and $R=R_{2}$. This result can be understood classically from the presence of snake orbits between regions $I$ and $I I$ 
and between regions $I I$ and $I I I$. Upon increasing $R$ to $R=70 \mathrm{~nm}$ the wave functions are mainly localized inside the hole of the ring $r<R-W / 2$ and only partially in the ring region $R-W / 2<r<R+W / 2$. For large $R$ the energy levels inside the hole of the ring are close to the LLs and $E_{(1,1)}>E_{(1,0)}>E_{(-1,0)}$. Because of that their order shown in the inset of Fig. 9(a), will change with increasing $R$.

We can also fix $R$ and plot the energy levels as a function of $W$ with $W$ in the range $0<W<2 R$. We show the results for $R=20 \mathrm{~nm}$ in Fig. 10 (a) and for $R=60 \mathrm{~nm}$ in Fig. 10(b). When $W$ is small (see Fig. 10(a)) the region $I I$ in Fig. 8 is narrow and the electron pushed out of this region. All electron states start from the LLs in region III (see the black dashed curves in Figs. 10(b) and (c)) and they will increase with increasing $W$. However, for large $W$ electrons can be localized in region $I I$ and as shown in 10(b) and (c) all levels converge to the LLs. The energy states for intermediate values of $W$ are below the LLs and are due to snake orbits localized around the boundaries.

The corresponding total wave functions for fixed $R=60 \mathrm{~nm}$ and three values of $W(W=20,60,100 \mathrm{~nm})$ are shown in Figs. 11(a)-(c). With increasing $W$ the electron probability to be in regions $I$ and $I I$ will increase. The insets in Figs. 11(b)-(d) shows the possible classical trajectories for different cases.

In order to show the non-quantization of the orbital momentum, we calculate the expectation value of the orbital momentum $L_{z}$ given by

$$
\left\langle L_{z}\right\rangle=\left\langle-\mathrm{i} \hbar \frac{\partial}{\partial \varphi}\right\rangle=m \hbar+\hbar \int|b(r)|^{2} d r .
$$

The numerical results for the magnetic ring profile shown in Fig. 8 are plotted in Fig. 12. As shown, when $R$ increases $\left\langle L_{z}\right\rangle$ tends to the quantized value $m \hbar$ while for intermediate values of $R$ we have $\left\langle L_{z}\right\rangle>m \hbar$. For small $R$ the difference $\left\langle L_{z}\right\rangle-m \hbar$ can be very large. Notice that, although our problem is circularly symmetric, $L_{z}$ is not a conserved quantity in graphene and the quantum number $m$ corresponds to the operator $J_{z}$ given by

$$
J_{z}=L_{z}+S_{z}
$$

where $S_{z}=\hbar \sigma_{z} / 2$ is the pseudospin. Using Eqs. (25) and (24) we obtain $\left\langle J_{z}\right\rangle=$ $(m+1 / 2) \hbar$.

In Fig. 13 we plot the bound states for a ring with $B_{1}=0$ and $B_{0}=B_{2}=B$, as function of $B$ in (a) and as function of an external magnetic field $B_{\text {ext }}$ in (b) with $B_{0}=5$ T. Notice that in Fig. 13(a) we have, for fixed $m$, several anti-crossings between levels with different $n$. These anti-crossings are a consequence of two different tendencies. On the one hand there exist electron states that are spread out over a large area, for which the $B=0$ ring area is a small perturbation, resulting in energies just below those of the LLs in a homogenous $B$ field. On the other hand, there are states that are predominantly localized in the $B=0$ ring area and their quantum confinement is strengthened with increasing $B$ and becomes equal to the one for a hard-wall in the limit of very large $B[31,32]$. When two of such states reach the same energy value they anti-cross. 
The situation is very different in Fig. 13(b) where the spectrum is shown as function of a homogenous applied magnetic field. Note that the spectrum is not invariant under the change $B_{\text {ext }} \rightarrow-B_{\text {ext }}$. For $\left|B_{\text {ext }}\right| \gg B_{0}$ we recover the $L L s$ for a homogenous field equal to $B_{\text {ext }}$. For intermediate values of $B_{\text {ext }}$ the degeneracy of the levels is lifted which is most pronounced for $B_{\text {ext }} \approx-B_{0}$. For intermediate $B_{\text {ext }}$ values the bound states are closely related to the classical snake orbits.
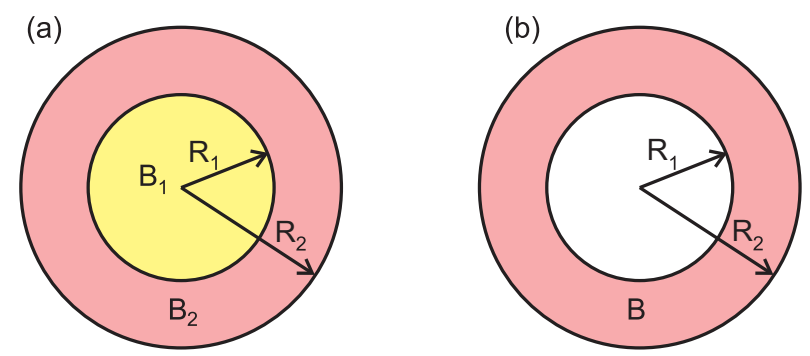

Figure 14. (a) A magnetic dot of radius $R_{1}$ in a field $B_{1}$ surrounded by an annulus of radius $R_{2}$ in a field $B_{2}$. (b) A magnetic ring profile.

\section{Magnetic field profiles of finite extent: quasi-bound states}

We now look for possible, long-living quasi-stationary states in graphene for the magnetic field profiles shown in Fig. 14. Such profiles can be created by a perpendicularly magnetized ferromagnetic disk placed above a 2DEG [6]. We expect that this is possible with graphene as well. To account for quasi-bound states one normally solves the timedependent Schrödinger equation which is a very complicated problem when compared to the standard eigenvalue problem. Here we follow the method presented in detail in Refs. $[4,20]$ and calculate the local density of states. The idea is to confine the electron in a large region of finite radius $R_{\infty} \gg R_{2}$, with its wave function vanishing at the border $\left(r=R_{\infty}\right)$, and treat the problem as a stationary one. The justification is that the result of measuring, e.g., a tunneling current directed perpendicular to the dot via a STM tip or the power absorption in near-field infrared spectroscopy, depends on the average value of the electron wave function in the dot. Therefore, we introduce the local density of states in a magnetic dot as

$$
\rho(E, m)=\frac{\pi k}{R} \sum_{n} \delta\left(E-E_{n, m}\right) \int_{0}^{\infty} r F(r)|\Psi(r)|^{2} d r
$$

which depends on the electron probability density and the aperture function $F(r)$ which characterizes the interaction of the electron with the measuring probe. From the fact that $\Delta E=k \Delta k=k \pi / R$, we can convert the sum over $n$ into an integral and write the final expression for the local density of states as

$$
\rho(E, m)=\frac{1}{2 \pi} \int_{0}^{\infty} r F(r)|\Psi(r)|^{2} d r
$$


The integral in Eq. (27) is sensitive to the probability of finding an electron in the dot and for a quasi-bound state it will exhibit a peak corresponding to the energy of this state. The width of the peak is proportional to the inverse of the lifetime of this quasi-bound state. For definiteness we use a Gaussian aperture function,

$$
F(r)=b R_{2}^{2} e^{-b r^{2}}, \quad b=R_{2}^{-2} \ln 10 .
$$

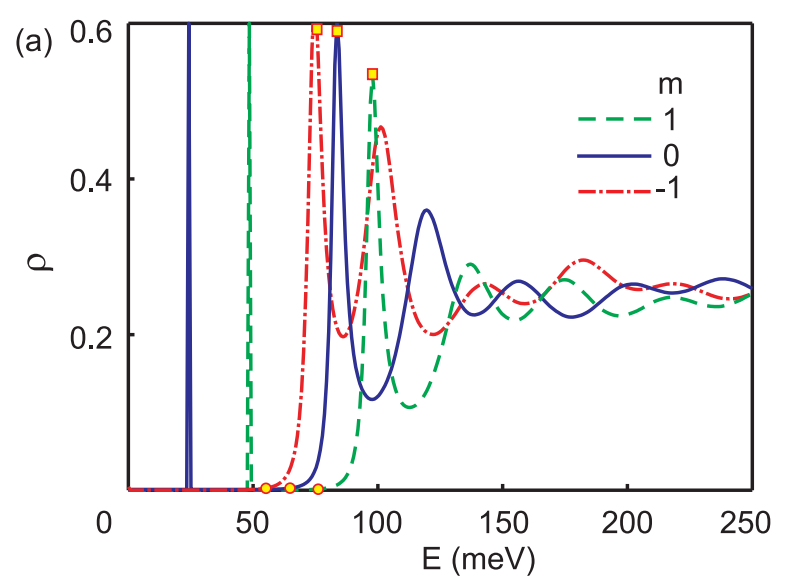

(b)
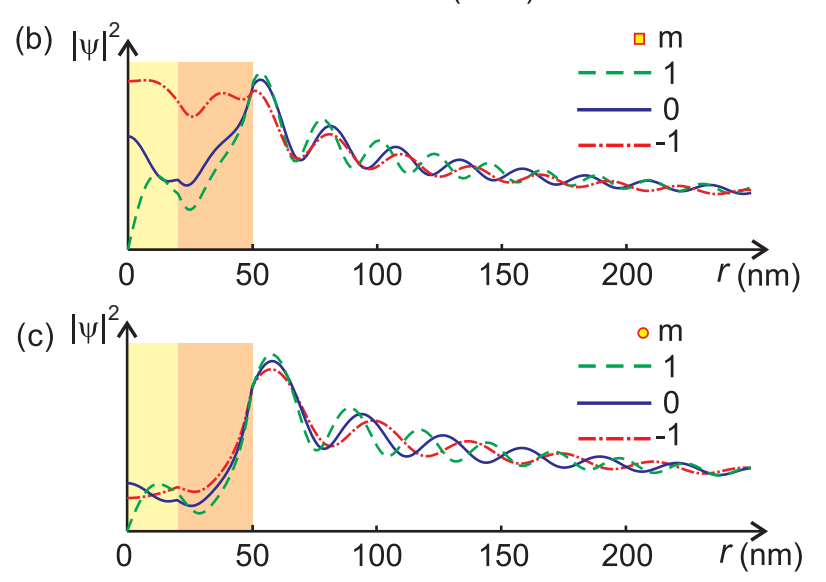

Figure 15. (a) Local density of states $\rho(E)$ versus energy for $m=0, \pm 1$ and $B_{2}=-B_{1}=5 \mathrm{~T}, R_{1}=20 \mathrm{~nm}$, and $R_{2}=50 \mathrm{~nm}$. The wave functions corresponding to the states marked by circles (squares) in (a) are shown in (b) and (c).

\subsection{Magnetic Dot}

We consider a magnetic dot in a perpendicular magnetic field $B_{1}$ surrounded by a field $B_{2}$ in a finite region outside it as shown in Fig. 14. Explicitly the field $B(r)$ is given by

$$
B(r)= \begin{cases}B_{1}, & 0<r<R_{1} \\ B_{2}, & R_{1}<r<R_{2} \\ 0, & R_{2}<r<\infty\end{cases}
$$


Graphene in inhomogeneous magnetic fields: Bound, quasi-bound, and scattering states18

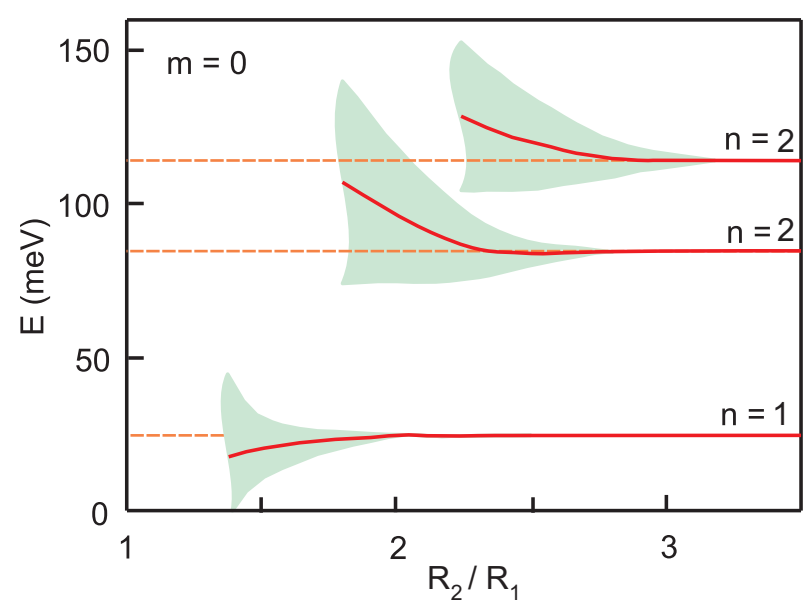

Figure 16. Quasi-bound states (red thick curves) with orbital momentum $m=0$ as a function of $R_{2} / R_{1}$ with $R_{1}=20 \mathrm{~nm}, B_{1}=-5 \mathrm{~T}$ (see Fig. 14(b)), and $B_{2}=5 \mathrm{~T}$. The light-green shaded area is a measure of the inverse lifetime of these states.

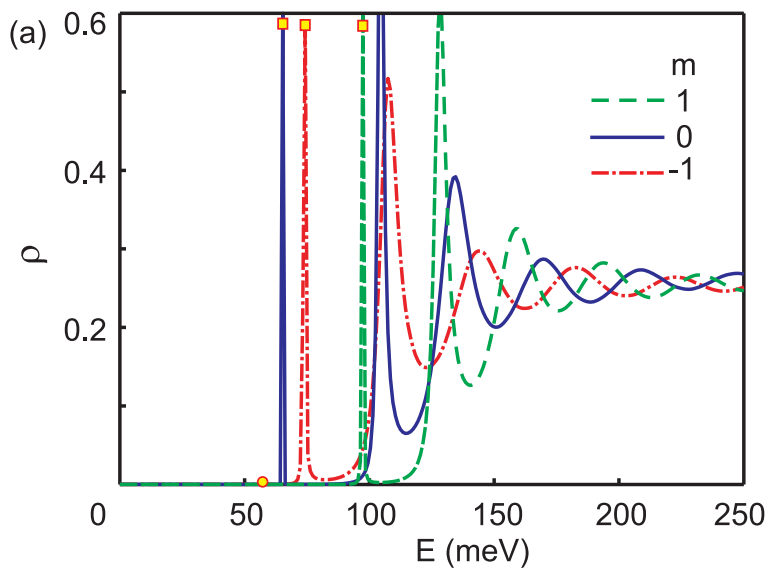

(b) $\mid$
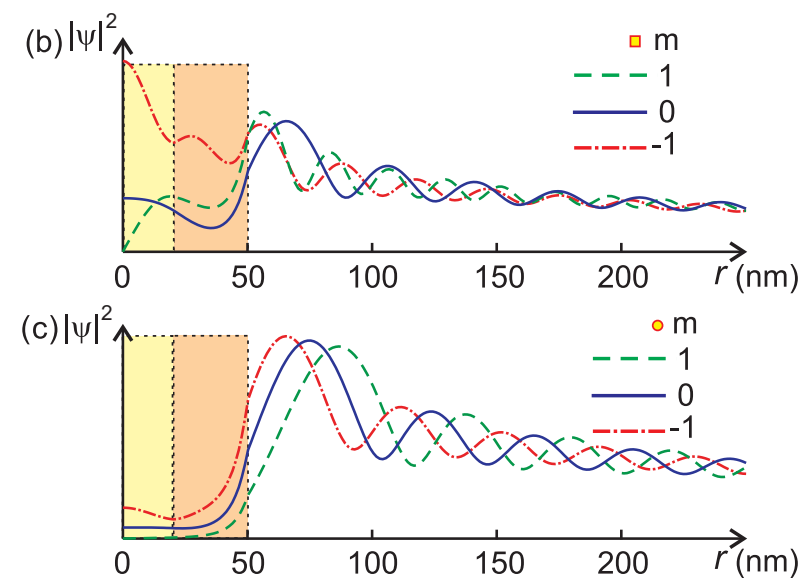

Figure 17. (a) Local density of states $\rho(E)$ versus energy for $m=-1,0,1, B_{1}=2.5$ $\mathrm{T}, B_{2}=5 \mathrm{~T}, R_{1}=20 \mathrm{~nm}$, and $R_{2}=50 \mathrm{~nm}$. The wave functions of the states marked by squares and circles in (a) are shown in (b) and (c), respectively. 


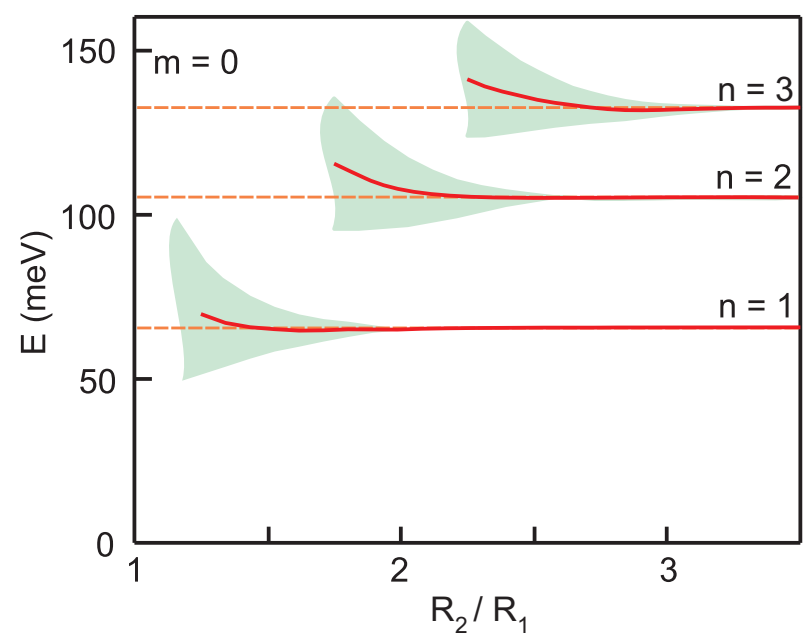

Figure 18. As in Fig. 16 but now for $B_{1}=2.5 \mathrm{~T}$ and $B_{2}=5 \mathrm{~T}$.

and the corresponding vector potential by

$$
A(r)=\frac{1}{r} \begin{cases}\gamma_{1} r^{2}, & 0<r<R_{1}, \\ \gamma_{2} r^{2}+\left(\gamma_{1}-\gamma_{2}\right) R_{1}^{2}, & R_{1}<r<R_{2}, \\ \gamma_{2} R_{2}^{2}+\left(\gamma_{1}-\gamma_{2}\right) R_{1}^{2}, & R_{2}<r<\infty .\end{cases}
$$

We proceed as in Sec. II and obtain Eq. (4) which we convert into two second-order differential equations for the components $a(r)$ and $b(r)$. The one for $b(r)$ is again given by Eq. (7). Explicitly, the solutions for $b(r)$ in the three different regions are, respectively,

$$
\begin{aligned}
& b_{1}(r)=A r^{|m+1|} e^{-\left|\gamma_{1}\right| r^{2} / 2} M\left(\alpha_{1}, \beta_{1}, z_{1}\right), \\
& b_{2}(r)=r^{|m+1|} e^{-\left|\gamma_{2}\right| r^{2} / 2}\left\{B M\left(\alpha_{2}, \beta_{2}, z_{2}\right)+C U\left(\alpha_{2}, \beta_{2}, z_{2}\right)\right\},
\end{aligned}
$$

and

$$
b_{3}(r)=G J_{\nu}(|E| r)+H Y_{\nu}(|E| r)
$$

with $\nu=s_{3}+m+1 . J_{\nu}(z)$ and $Y_{\nu}(z)$ are the Bessel and modified Bessel functions, respectively. The solutions for $a(r)$ are obtained from Eq. (4). The components $a(r)$ and $b(r)$ obey the following boundary conditions at $r=R_{1}$ and $r=R_{2}: a_{1}\left(R_{1}\right)=a_{2}\left(R_{1}\right)$, $b_{1}\left(R_{1}\right)=b_{2}\left(R_{1}\right), a_{2}\left(R_{2}\right)=a_{3}\left(R_{2}\right)$ and $b_{2}\left(R_{2}\right)=b_{3}\left(R_{2}\right)$. The condition that the wave function has to vanish for $r \rightarrow \infty$ leads to $G^{2}+H^{2}=1$.

In Fig. 15 we plot the local density of states for a finite dot with antiparallel magnetic fields inside and outside it for two different dot sizes (the structure shown in Fig. 14(a)). Now the energy states are broadened, i. e., they have a finite lifetime and no bound states exist. The sharp peaks for $m=0$ and $m=1$ in Fig. 15(a) are reminiscent of the previous bonding and anti-bonding states of the $n=1$ split level. We also plot the total wave function for three different energies indicated by squares and circles, respectively in Figs. 15(b) and (c). We see that when the energy is close to that of a quasi-bound state the probability of finding the electron inside the dot becomes 
large. Such states are also called resonant states. The energy of these quasi-bound states for angular momentum $m=0$, i. e., for s-wave scattering, are shown in Fig. 16 and, as expected, for $R_{2} \gg R_{1}$ the quasi-bound states will be close to the energy of the LLs (shown by the horizontal dashed lines in Fig. 16). The width of the resonances in Fig. 15(a), proportional to the inverse lifetime of these quasi-bound states, is shown by the shaded areas in Fig. 16.

We also show the numerical results for a magnetic dot, with parallel magnetic fields inside and outside it, in Figs. 17 and 18. Notice that qualitatively the results are rather similar except that in Fig. 16 the lowest energy bound state is below the LL result for $R_{2} / R_{1}<2$ while in Fig. 18 this is above the LL.

\subsection{Magnetic Ring}
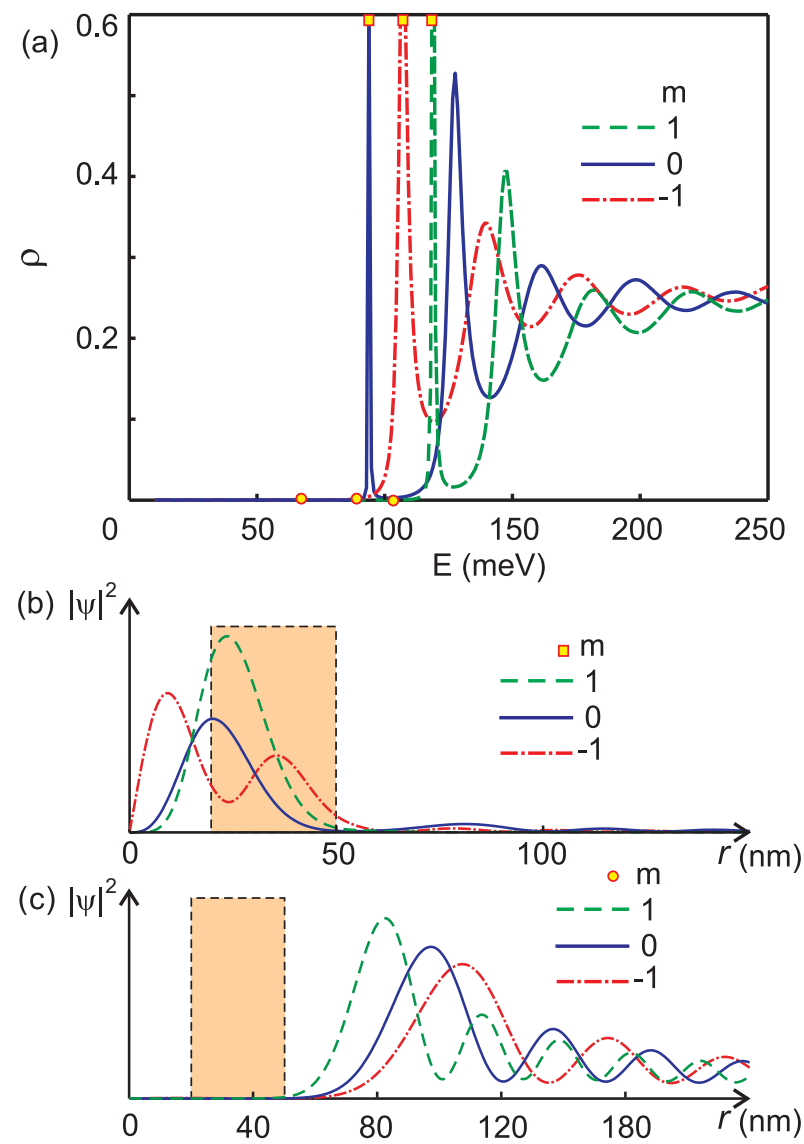

Figure 19. (a) Local density of states $\rho(E)$ versus energy for $m=-1,0,1$ for the magnetic ring profile shown in Fig. $14(\mathrm{~b})$ with $B=5 \mathrm{~T}, R_{1}=20 \mathrm{~nm}$ and $R_{2}=50$ $\mathrm{nm}$. The wave functions corresponding to the states marked by squares and circles in (a) are shown in (b) and (c), respectively.

Next we consider the magnetic ring shown in Fig. 14(b); its profile can be obtained from the previous one by setting $B_{1}=0$. The new magnetic profile is 
$B(\mathbf{r})=B \Theta\left[\left(R_{2}-r\right)\left(r-R_{1}\right)\right]$ and the corresponding vector potential profile is given by

$$
A(r)=\frac{\gamma}{r} \begin{cases}R_{1}^{2}, & 0<r<R_{1} \\ r^{2}, & R_{1}<r<R_{2}, \\ R_{2}^{2}, & R_{2}<r<\infty .\end{cases}
$$

The solutions for $b(r)$ in the three different regions are, respectively,

$$
b_{1}(r)=A J_{\nu_{1}}(|E| r)
$$

with $\nu_{1}=s_{1}+m+1$ and $s_{1}=\gamma R_{1}^{2}$,

$$
b_{2}(r)=r^{|m+1|} e^{-|\gamma| r^{2} / 2}\{B M(\alpha, \beta, z)+C U(\alpha, \beta, z)\}
$$

and

$$
b_{3}(r)=G J_{\nu_{2}}(|E| r)+H Y_{\nu_{2}}(|E| r),
$$

with $\nu_{2}=s_{3}+m+1$ and $s_{3}=\gamma R_{2}^{2}$.

In Fig. 19(a) we plot the numerically evaluated local density of states for three different quantum numbers $m$. The total wave function is plotted for two different cases. When the energy is close to that of a quasi-bound state (b) the electron is localized inside the ring, cf. Fig. 19(b). When it is not, cf. Fig. 19(c), the electron does not penetrate the ring area. The results for the energy and width of the resonant states are qualitatively very similar with those of Fig. 18 and are therefore not shown.

\section{Magnetic dot with $\langle B\rangle=0$}

A magnetic dot with zero average magnetic field $\left\langle B_{z}\right\rangle=0$ is a structure that can be realized experimentally by depositing, e. g., Dy micromagnets on top of graphene with an insulating layer in-between to prevent electrical contact; this was previously realized on top of a $G a A s / A l_{x} G a_{1-x} A s$ heterostructure[27, 28]. We model the magnetic field profile by a simple step as depicted in Fig. 20. The field $B(\mathbf{r})$ is

$$
B(\mathbf{r})=\left\{\begin{array}{lc}
B_{1}, & r<R_{1} \\
B_{2}, & R_{1}<r<R_{2} . \\
0, & r>R_{2}
\end{array}\right.
$$

The condition $\left\langle B_{z}\right\rangle=0$ is satisfied for $B_{2}=-B_{1} /\left(R_{2}^{2} / R_{1}^{2}-1\right)$. The solutions in the three different regions are the same as those given by Eqs. (31), (32), and (33).

Such a system can support only quasi-bound states. The numerical results for the local density of states are shown in Fig. 21 for two different sizes of the magnetic dot and different quantum numbers $m$. In contrast with the previous cases we see that for $m \geq 0$ there are pronounced peaks close to zero energy. When the ratio $R_{2} / R_{1}$ increases these peaks become sharper indicating long-living, quasi-bound states. Later on we will show that these states give rise to a pronounced structure in the magnetoresistance and the Hall resistance. The wave functions corresponding to the high local density of states are shown in Fig. 21(c) and those at the energy of the first LL in Fig. 21(b). 

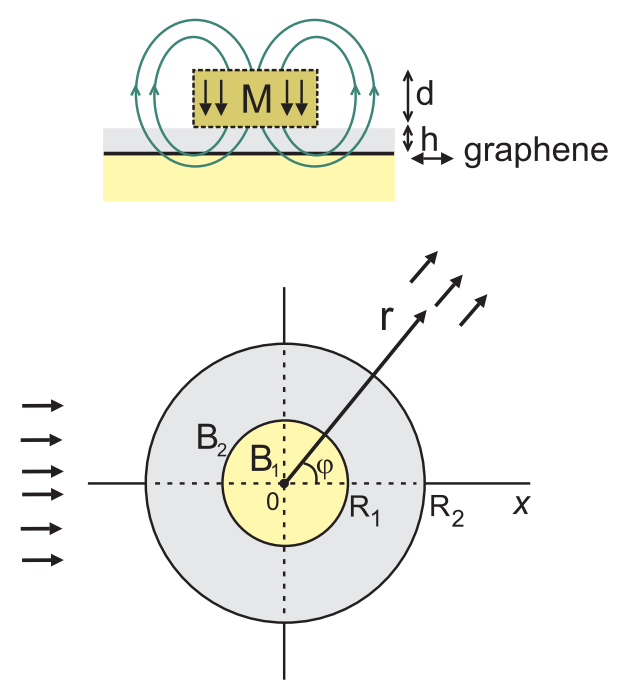

Figure 20. Field profile for a magnetic dot with total zero magnetic flux (lower panel). The upper panel shows a perpendicularly magnetized ferromagnetic disk of thickness $d$ held a distance $h$ above graphene that could lead to such a profile.

\subsection{Zero energy state for a finite magnetic dot}

The solution presented in Sec. 3.1 should be modified in the case of a finite magnetic dot. Here we have three regions and the solution outside the magnetic dot,where $B$ vanishes, is given by

$$
a(r) \sim r^{m+s_{3}}
$$

and

$$
b(r) \sim r^{-m-1-s_{3}} .
$$

In order to have a zero-energy state we should have $m \leq-s_{3}$ for the component $a(r)$ and $m \geq-s_{3}$ for the component $b(r)$. From the fact that $a(r)$ is converging in the first region for $m \geq-s_{1}$ and $b(r)$ for $m \leq-s_{1}-1$, the zero-energy states for the previously discussed structures, are as follows.

1) Magnetic dot of finite size (cf. Fig. 14(a)). In this case $s_{1}=0$ and $s_{3}=\gamma_{2} R_{2}^{2}+\left(\gamma_{1}-\gamma_{2}\right) R_{1}^{2}=\Phi / \phi_{0}$, where $\Phi$ is the total magnetic flux with $\phi_{0}=\hbar / e$ the flux quantum. Then $a(r)$ and $b(r)$ should satisfy the conditions

$$
\left\{\begin{array}{l}
a(r) \rightarrow 0 \leq m<-\left[\Phi / \phi_{0}\right] \\
b(r) \rightarrow-\left[\Phi / \phi_{0}\right]-1<m \leq-1
\end{array}\right.
$$

where $\left[\Phi / \phi_{0}\right]=n$ is the largest integer smaller than $\Phi / \phi_{0}$. These two conditions cannot be satisfied at the same time. Then $a(r)=0$ for $\Phi>0$ and the second condition is true. In order to have zero-energy states we have to limit the angular momentum $m$ for the second component as $-\left[\Phi / \phi_{0}\right] \leq m \leq-1$. For $\Phi / \phi_{0}<0$ the first condition is true and we have to choose $m$ for the first component in the range $0 \leq m<-\left[\Phi / \phi_{0}\right]$. Then we have a $n$ times degenerate, zero-energy level. 
2) Magnetic ring of finite size (cf. Fig. 14(b)). In this case we have $s_{1}=\gamma R_{1}^{2}=$ $\Phi_{1} / \phi_{0}$ and $s_{3}=\gamma R_{2}^{2}=\Phi_{2} / \phi_{0}$. Repeating the procedure above we find that zero-energy states exist under the condition $-\left[\Phi_{2} / \phi_{0}\right] \leq m \leq-\left[1+\Phi_{1} / \phi_{0}\right]$.

3) Magnetic dot with $\left\langle B_{z}\right\rangle=0$, (cf. Fig. 20). Because the average magnetic field is zero, the total flux is zero and we do not have any zero-energy states. It is also possible to show that a zero-energy solution for a finite-size magnetic dot is not possible because $s_{3}=0$, and $\left[\Phi / \phi_{0}\right]=0$ which leads to $0 \leq m \leq-1$ which clearly can not be satisfied.

\subsection{Elastic scattering}

Next we concentrate on the scattering problem of Dirac electrons by a circularly symmetric magnetic dot with $\langle B\rangle=0$. The layout of the problem is shown in Fig. 20 . We assume that electrons are coming from $-\infty$, along the $x$ axis, and calculate the probability for them to be scattered by the dot and deflected by an angle $\varphi$. The free-electron wave function is

$$
\Psi_{0}(\boldsymbol{r})=\frac{1}{\sqrt{2}}\left(\begin{array}{l}
1 \\
1
\end{array}\right) \mathrm{e}^{\mathrm{i} k x}
$$

with $k>0$. It describes a homogeneous electron flow with density $\rho=1$ and current $\boldsymbol{J}=(1,0)$ in units of $e v_{F}$.

In standard scattering theory the wave function far away from the scatterer is presented as a sum of two parts,

$$
\Psi(\boldsymbol{r}) \approx \Psi_{0}(\boldsymbol{r})+\frac{f(\varphi)}{\sqrt{r}} \mathrm{e}^{\mathrm{i} k r}
$$

where the first part corresponds to the incoming electron while the second part, called scattering amplitude, has two components as the wave function itself,

$$
f=\left(\begin{array}{c}
f_{1} \\
f_{2}
\end{array}\right) .
$$

The macroscopic scattering characteristic is the differential cross section which is defined as the number of electrons scattered into the angle element $d \varphi$ per unit time. It is given by the electron velocity multiplied by the arc length $r d \varphi$ of a rather large-radius circle, where this electron flow is measured. Having in mind the definition $\boldsymbol{J}=\Psi^{+} \sigma \Psi$ we define the differential cross section $d \sigma(\varphi)$ by

$$
d \sigma(\varphi)=P(\varphi) d \varphi=f^{+}(\varphi)(\sigma \cdot \boldsymbol{n}) f(\varphi) d \varphi .
$$

Here $\boldsymbol{n}$ stands for the unit vector perpendicular to the above mentioned circle, $P(\varphi)$ is given by

$$
P(\varphi)=\mathrm{e}^{\mathrm{i} \varphi} a b^{*}+\mathrm{e}^{-\mathrm{i} \varphi} a^{*} b,
$$

and the total cross section by

$$
\sigma_{t o t}=\int_{0}^{2 \pi} P(\varphi) d \varphi
$$


Graphene in inhomogeneous magnetic fields: Bound, quasi-bound, and scattering states 24

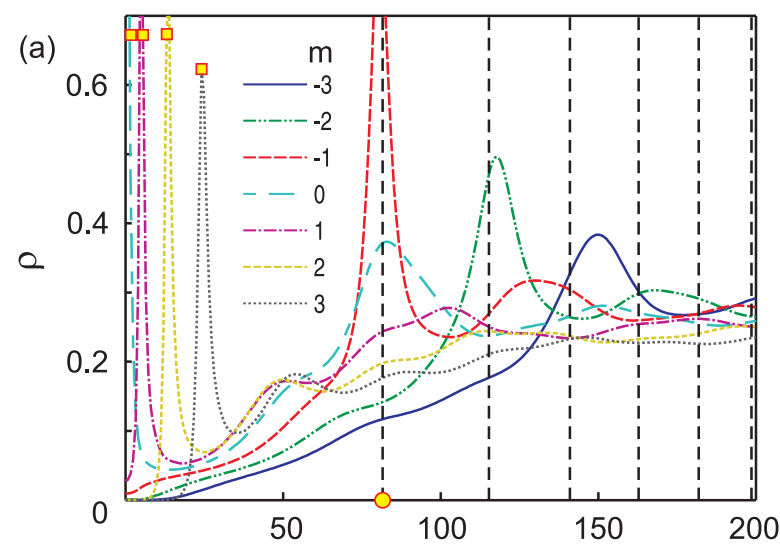

(b)

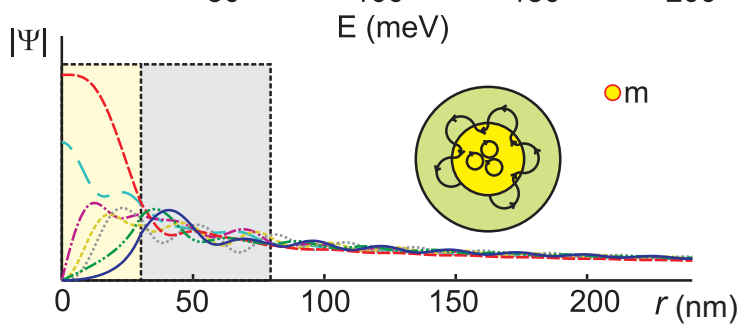

(c)

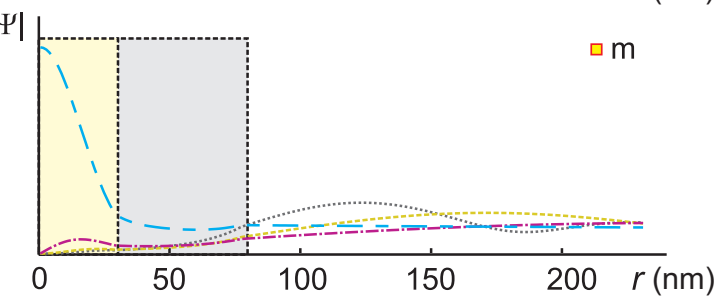

Figure 21. (a) Local density of states for $m=0, \pm 1, \pm 2, \pm 3$ and the magnetic field profile shown in Fig. 20 with $B_{1}=-5 T$ and $\left(R_{1}, R_{2}\right)=(30,80) \mathrm{nm}$. The vertical dashed lines show the position of the LLs. (b) Total wave function for the first LL, indicated by a circle in (a) for different values of $m$. The inset shows the electron trajectory. (c) As in (b) for the peaks indicated by squares in (a).

We express the solution outside the dot as a combination of Bessel functions

$$
a(r)=w_{n}\left\{\cos \delta_{m} \cdot J_{m}(k r)+\sin \delta_{m} \cdot Y_{m}(k r)\right\} .
$$

Then the components of the eigenfuction are related by

$$
b(r)=-\frac{1}{E}\left\{\frac{d}{d r}-\frac{m}{r}\right\} a(r)=w_{n}\left\{\cos \delta_{m} \cdot J_{m+1}(k r)+\sin \delta_{m} \cdot Y_{m+1}(k r)\right\} .
$$

This leads to the expansions

$$
\begin{aligned}
& \psi_{1}(r, \varphi)=\sum_{m=-\infty}^{\infty} \mathrm{e}^{\mathrm{i} m \varphi} w_{n}\left\{\cos \delta_{m} \cdot J_{m}(k r)+\sin \delta_{m} \cdot Y_{m}(k r)\right\} \\
& \psi_{2}(r, \varphi)=\mathrm{i} \sum_{m=-\infty}^{\infty} \mathrm{e}^{\mathrm{i}(m+1) \varphi} w_{n}\left\{\cos \delta_{m} \cdot J_{m+1}(k r)+\sin \delta_{m} \cdot Y_{m+1}(k r)\right\}
\end{aligned}
$$

for the components of the wave function $\psi(r, \varphi)$. The coefficients $w_{n}$ and $\delta_{m}$ are determined by the boundary conditions at $R_{1}, R_{2}$ and the wave function (42). 
Graphene in inhomogeneous magnetic fields: Bound, quasi-bound, and scattering states25

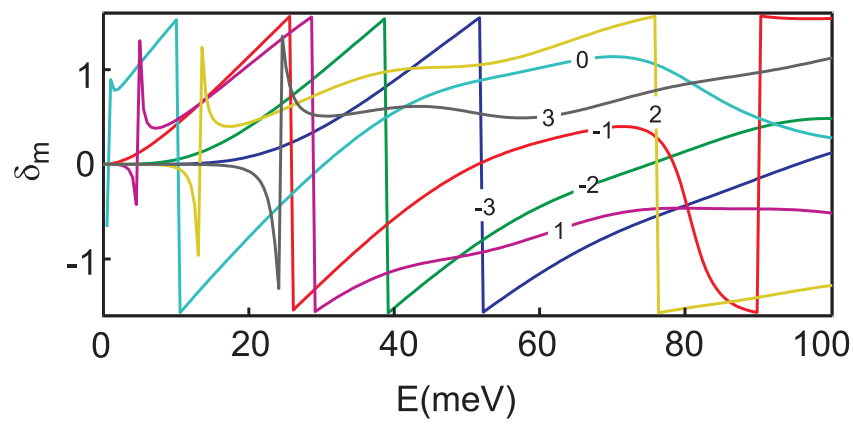

Figure 22. The phase shift $\delta_{m}$ as a function of the energy for $m=0, \pm 1, \pm 2, \pm 3$, $B_{1}=-5 T$, and $\left(R_{1}, R_{2}\right)=(30,80) \mathrm{nm}$.

For $r \rightarrow \infty$ the asymptotic expressions of the Bessel functions are $J_{m}(k r) \approx$ $(c / \sqrt{r}) \cos \Delta_{m}$ and $Y_{m}(k r) \approx(c / \sqrt{r}) \sin \Delta_{m}$, with $\Delta_{m}=k r-\pi m / 2-\pi / 4$ and $c=\sqrt{2 / \pi k}$. They enable us to present Eqs. (50) and (51) in the asymptotic region as

$$
\begin{aligned}
& \psi_{1}(\boldsymbol{r})=\frac{c}{\sqrt{r}} \sum_{m=-\infty}^{\infty} w_{m} \mathrm{e}^{\mathrm{i} m \varphi} \cos \left(\Delta_{m}-\delta_{m}\right), \\
& \psi_{2}(\boldsymbol{r})=\mathrm{i} \frac{c}{\sqrt{r}} \sum_{m=-\infty}^{\infty} w_{m} \mathrm{e}^{\mathrm{i}(m+1) \varphi} \cos \left(\Delta_{m+1}-\delta_{m}\right) .
\end{aligned}
$$

These expansions must agree with the asymptotic form of Eq. (42). For this purpose we write the exponential in Eq. (42) as

$$
\mathrm{e}^{\mathrm{i} k r \cos \varphi}=\sum_{m=-\infty}^{\infty} \mathrm{i}^{m} \mathrm{e}^{\mathrm{i} m \varphi} J_{m}(k r) \approx \frac{c}{2 \sqrt{r}} \sum_{m=-\infty}^{\infty} \mathrm{i}^{m} \mathrm{e}^{\mathrm{i} m \varphi}\left(\mathrm{e}^{\mathrm{i} \Delta_{m}}+\mathrm{e}^{-\mathrm{i} \Delta_{m}}\right) .
$$
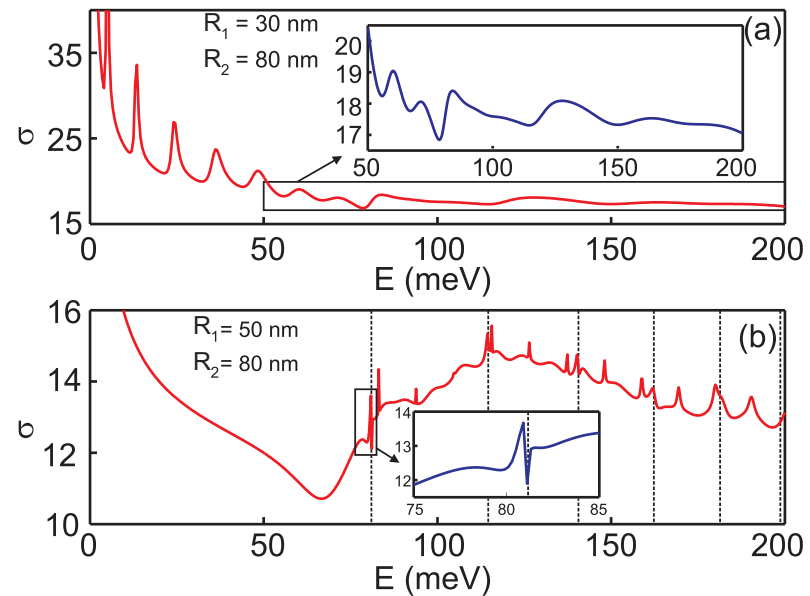

Figure 23. Total cross section for $B_{1}=-5 T$ with $\left(R_{1}, R_{2}\right)=(30,80) \mathrm{nm}$ in (a) and $\left(R_{1}, R_{2}\right)=(50,80) \mathrm{nm}$ in $(\mathrm{b})$. 
Graphene in inhomogeneous magnetic fields: Bound, quasi-bound, and scattering states26

First, we compare Eq. (52) with the first wave function component (50) by rewriting Eq. (52) as

$$
\psi_{1}(\boldsymbol{r})=\frac{c}{2 \sqrt{r}} \sum_{m=-\infty}^{\infty} w_{m} \mathrm{e}^{\mathrm{i} m \varphi}\left\{\mathrm{e}^{\mathrm{i}\left(\Delta_{m}-\delta_{m}\right)}+\mathrm{e}^{-\mathrm{i}\left(\Delta_{m}-\delta_{m}\right)}\right\} .
$$

The coefficients of the exponents representing the incoming wave (the second term inside the curly brackets) must be equal. This gives

$$
w_{m}=\mathrm{i}^{m} \mathrm{e}^{-\mathrm{i} \delta_{m}} .
$$

Subtracting the asymptotic from Eq. (55) gives the first component of the scattered wave function

$$
\psi_{1}^{(\text {scatt })}(\boldsymbol{r})=\frac{c}{2 \sqrt{r}} \sum_{m=-\infty}^{\infty} \mathrm{i}^{m} \mathrm{e}^{\mathrm{i} m \varphi} \mathrm{e}^{\mathrm{i} \Delta_{m}}\left\{\mathrm{e}^{-2 \mathrm{i} \delta_{m}}-1\right\} .
$$

Now we have to check whether the second wave function component is correct in the asymptotic region. The second sum representing the incoming wave has to be the same as the first component. This second component can be written as

$$
\psi_{2}(\boldsymbol{r})=\frac{c}{2 \sqrt{r}} \sum_{m=-\infty}^{\infty} \mathrm{i}^{m+1} \mathrm{e}^{-\mathrm{i} \delta_{m}} \mathrm{e}^{\mathrm{i}(m+1) \varphi}\left\{\mathrm{e}^{\mathrm{i}\left(\Delta_{m+1}-\delta_{m}\right)}+\mathrm{e}^{-\mathrm{i}\left(\Delta_{m+1}-\delta_{m}\right)}\right\} .
$$
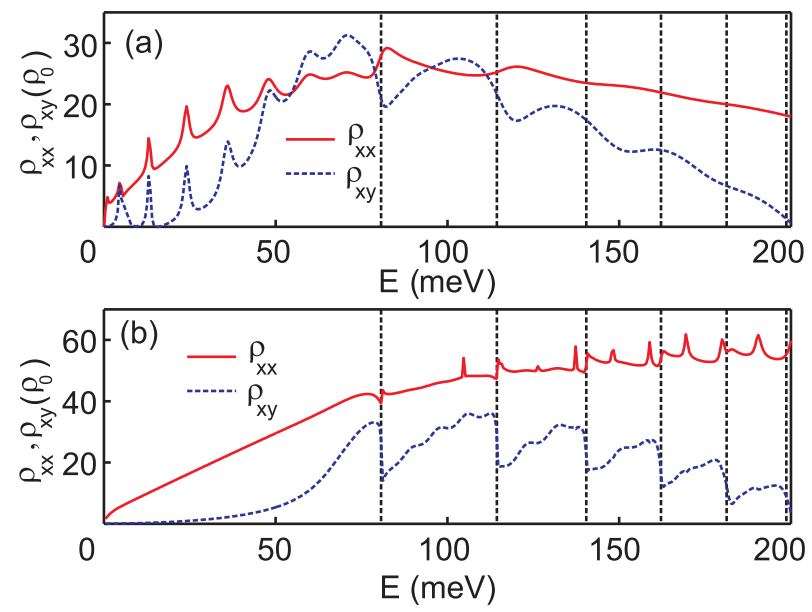

Figure 24. The magnetoresistance (red, solid curve) and Hall resistance (blue, dashed curve) as a function of the Fermi energy $E_{F}$ for $B_{1}=-5 T$, (a) $\left(R_{1}, R_{2}\right)=(30,80) \mathrm{nm}$ and (b) $\left(R_{1}, R_{2}\right)=(50,80) \mathrm{nm}$.

We can rewrite the scattered part of the wave function components in the following final form:

$$
\begin{aligned}
& \psi_{1}^{(\text {scatt })}(\boldsymbol{r})=\frac{c}{2 \sqrt{r}} \sum_{m=-\infty}^{\infty} \mathrm{i}^{m} \mathrm{e}^{\mathrm{i} m \varphi} \mathrm{e}^{\mathrm{i} \Delta_{m}}\left\{\mathrm{e}^{-2 \mathrm{i} \delta_{m}}-1\right\} \\
& \psi_{2}^{(\text {scatt })}(\boldsymbol{r})=\frac{c}{2 \sqrt{r}} \sum_{m=-\infty}^{\infty} \mathrm{i}^{m} \mathrm{e}^{\mathrm{i} m \varphi} \mathrm{e}^{\mathrm{i} \Delta_{m}}\left\{\mathrm{e}^{-2 \mathrm{i} \delta_{m-1}}-1\right\} .
\end{aligned}
$$


This enables us to write the components of the scattering amplitude (44) in the form

$$
\begin{aligned}
& f_{1}=\mathrm{e}^{-\mathrm{i} \pi / 4} \frac{c}{2 \sqrt{r}} \sum_{m=-\infty}^{\infty} \mathrm{e}^{\mathrm{i} m \varphi}\left\{\mathrm{e}^{-2 \mathrm{i} \delta_{m}}-1\right\}, \\
& f_{2}=\mathrm{e}^{-\mathrm{i} \pi / 4} \frac{c}{2 \sqrt{r}} \sum_{m=-\infty}^{\infty} \mathrm{e}^{\mathrm{i} m \varphi}\left\{\mathrm{e}^{-2 \mathrm{i} \delta_{m-1}}-1\right\},
\end{aligned}
$$

the differential cross section (46) as

$$
P(\varphi)=\frac{4}{\pi k} \sum_{m, m^{\prime}=-\infty}^{\infty} \mathrm{e}^{\mathrm{i}\left[\left(m-m^{\prime}\right) \varphi-\left(\delta_{m}-\delta_{m^{\prime}}\right)\right]} \sin \delta_{m} \sin \delta_{m^{\prime}},
$$

and the total cross section as

$$
\sigma_{t o t}(E)=\frac{8}{k} \sum_{m=-\infty}^{\infty} \sin ^{2} \delta_{m}(E)
$$

For a random distribution of dilute, non-overlapping magnetic scatterers the zerotemperature magnetoresistance and Hall resistance are given by [30]

$$
\begin{aligned}
& \rho_{x x} / \rho_{0}=\int_{-\pi}^{\pi} d \varphi w(k, \varphi)(1-\cos \varphi)=\sum_{m=-\infty}^{\infty} 4 \sin ^{2}\left(\delta_{m}-\delta_{m+1}\right), \\
& \rho_{x y} / \rho_{0}=\int_{-\pi}^{\pi} d \varphi w(k, \varphi) \sin \varphi=\sum_{m=-\infty}^{\infty} 2 \sin \left[2\left(\delta_{m}-\delta_{m+1}\right)\right],
\end{aligned}
$$

with $\rho_{0}=\left(1 / 4 \pi^{2}\right)\left(n_{0} / n_{e}\right)\left(\hbar / e^{2}\right)$ where $n_{e}$ is the electron concentration, $n_{0}$ the concentration of magnetic scatterers, and $w(k, \varphi)=k P(\varphi)$ the probability for an electron with wave vector $k$ to be scattered by an angle $\varphi$.

In Fig. 21 we show the local density of states as a function of energy for $\left(R_{1}, R_{2}\right)=(30,80) \mathrm{nm}$ with $B_{1}=5 \mathrm{~T}$. The resonances are near the position of the LLs as shown by the vertical dashed lines. For $R_{1}$ small we have quasi-bound states between the $E=0$ and the first LLs for $m>0$. These levels move towards $E=0$ if we increase $R_{1}$. The phase shifts $\delta_{m}$ are shown in Fig. 22; resonances occur for energies with jumps in $\delta_{m}$.

In Figs. 23(a) and (b) we plot the total cross section as a function of $E$. Classically and in two dimensions this is equal to the total diameter of the magnetic inhomogeneity $\sigma=2 R_{b}$. The resonances shown up as small peaks, corresponding to the quasi-bound states of Fig. 21(a). Notice that the peaks in Fig. 23(a) for small energies $(E<50)$ are absent in Fig. 23(b). The difference comes from the fact that when we increase $R_{1}$ the structure behaves more or less like a finite size magnetic dot. As discussed in Sec. 5, zero-energy states exists for $0 \leq m<-\left[\Phi / \phi_{0}\right]$. Here all bound states in the finite-size magnetic dot become quasi-bound states because of the zero total magnetic flux. For $R_{1}$ large these levels are close to the $E=0$ level whereas for $R_{1}$ small these quasi-bound states move away from the $E=0$ level.

The corresponding magnetoresistance and Hall resistance are shown in Figs. 24(a) and (b) for the same dot structure. We found different behaviors in two energy regimes: 1) For $0<E<E_{1}$, where $E_{1}$ is the energy of the first LL, i. e. $E_{1} \approx 81 \mathrm{meV}$, 
all resonances result from the quasi-bound states shown in Fig. 21(a). This model is comparable with the magnetic dot discussed in section 3. As shown in Fig. 3 in the case of opposite sign of magnetic field in the inner and outer region the zero energy levels split for $m>0$ and now all these split levels become quasi-bound states as shown in Fig. 21(a). These resonances are not found in a normal 2DEG [7] and are unique to graphene. 2) For $E>E_{1}$ there are two types of resonances: first those which occur at the energy of the LLs of the inner core of the magnetic-field profile and second those corresponding to quasi-bound snake-orbit states that are located around the interface between the $B>0$ and $B<0$ regions.

\section{Conclusions}

We studied the bound, quasi-bound, and scattered states for electrons in graphene in the presence of circular inhomogeneous magnetic field profiles. The corresponding spectra were investigated as a function of the size and radii, the strength of the inhomogeneous magnetic field and of a uniform background field, and the angular momentum quantum number. More explicitly, we studied the following.

We considered the eigenvalue problem of a model quantum magnetic dot in the presence of a background field $B_{2}$, cf. Fig. 1, and a homogeneous magnetic field $B_{1}$ restricted in a finite, circular region of radius $R$. Both $B_{1}$ and $B_{2}$ are perpendicular to the plane of the dot. We studied the bound states for two different cases: 1) when the inner and outer magnetic fields have different sign $\left(B_{1}<0\right.$ and $\left.B_{2}>0\right)$ and 2) when both inner and outer magnetic fields have the same sign. In case 1) we found that the energy levels are split into bonding and anti-bonding states.

Bound states for of a magnetic ring in the presence of an external homogenous magnetic field were also studied.

Magnetic dot profiles of finite extent behave very differently. In this case the magnetic field fails to confine electrons. Although in such a magnetic field profile no confined states were found, quasi-bound sates with a finite lifetime are present. We presented a study of the local density of states and its dependence on the dot radius. Peaks in the local density of state are broadened and the width corresponds to the inverse lifetime of the quasi-bound state.

Special attention was paid to the zero-energy states for all different magnetic dot and ring profiles and we showed that zero-energy bound states exist when the magnetic flux is non-zero.

Finally, we considered a magnetic dot with $\left\langle B_{z}\right\rangle=0$ and the scattering of electrons on it. We calculated the local density of states, the magnetoresistance and the Hall resistance. All the zero-energy states that exist for finite size magnetic dots become quasi-bound states. These quasi-bound states are located close to $E=0$ and are found only for Dirac electrons. This results into two different energy regions in the magnetoresistance and the Hall resistance. They differ by the presence of sharp peaks due to resonant scattering involving these quasi-bound states. 
Graphene in inhomogeneous magnetic fields: Bound, quasi-bound, and scattering states29

\section{ACKNOWLEDGEMENTS}

This work was supported by the European Science Foundation (ESF) under the EUROCORES Program EuroGRAPHENE, the Canadian NSERC Grant No. OGP0121756, and the Belgian Science Policy (IAP). We acknowledge discussions and correspondence with Prof. A. Matulis.

\section{References}

[1] A. H. Castro Neto, F. Guinea, N. M. R. Peres, K. S. Novoselov, and A. K. Geim, Rev. Mod. Phys. 81, 109 (2009); D. S. L. Abergel, V. Apalkov, J. Berashevich, K. Ziegler, and T. Chakraborty, Adv. Phys. 59, 261 (2010).

[2] O. Klein, Z. Phys. 53, 157 (1929); M. I. Katsnelson, K. S. Novoselov, and A. K. Geim, Nature Physics 2, 620 (2006).

[3] Hong-Yi Chen, V. Apalkov, and T. Chakraborty, Phys. Rev. Lett. 98, 186803 (2007).

[4] A. Matulis and F. M. Peeters, Phys. Rev. B 75, 125429 (2007).

[5] P. Hewageegana and V. Apalkov, Phys. Rev. B 77, 245426 (2008).

[6] F. M. Peeters and A. Matulis, Phys. Rev. B 48, 15166 (1993).

[7] J. Reijniers, F. M. Peeters, and A. Matulis, Phys. Rev. B 64, 245314 (2001).

[8] A. De Martino, L. Dell' Anna, and R. Egger, Phys. Rev. Lett. 98, 066802 (2007).

[9] M. Ramezani Masir, P. Vasilopoulos, A. Matulis, and F. M. Peeters, Phys. Rev. B 77, 235443 (2008).

[10] S. Park and H. S. Sim, Phys. Rev. B 77, 075433 (2008).

[11] L. Oroszlany, P. Rakyta, A. Kormanyos, C. J. Lambert, and J. Cserti, Phys. Rev. B 77, 081403(R) (2008).

[12] T.K. Ghosh, A. De Martino, W. Häusler, L. Dell'Anna, and R. Egger, Phys. Rev. B 77, 081404(R) (2008).

[13] F. Zhai and K. Chang, Phys. Rev. B 77, 113409 (2008).

[14] Hengyi Xu, T.Heinzel, M. Evaldsson, and I. V. Zozoulenko, Phys. Rev. B 77, 245401 (2008).

[15] C. M. Lee, R. C. H. Lee, W. Y. Ruan, and M. Y. Chou, Appl. Phys. Lett. 96, 212101 (2010).

[16] D. Wang and G. Jin, Phys Lett. A 373, 4082 (2009).

[17] A. Zazunov, A. Kundu, A. Hutten, and R. Egger, Phys. Rev. B 82, 155431 (2010).

[18] Vitor M. Pereira and Antonio H. Castro Neto, Phys. Rev. Lett. 103, 046801 (2009) .

[19] F. Guinea, M. I. Katsnelson, and M. A. H. Vozmediano, Phys. Rev. B 77, 075422 (2008).

[20] M. Ramezani Masir, A. Matulis, and F. M. Peeters, Phys. Rev. B 79, 155451 (2009).

[21] I. S. Gradshteyn and I. M. Ryzhik, Tables of Integrals, Series, an Products, (Academic Press, New York, 2000), p. 699.

[22] M. I. Katsnelson, Mater. Today 10, 20 (2007).

[23] V. P. Gusynin and S. G. Sharapov, Phys. Rev. B 71, 125124 (2005); P. M. Krstajić and P. Vasilopoulos, Phys. Rev. B 83, 075427 (2011).

[24] D. B. Melrose and A. J. Parle, Ausl. J. Phys., 36, 755 (1983).

[25] G. W. Semenoff, Phys. Rev. Lett. 53, 2449 (1984).

[26] K. S. Novoselov, A. K. Geim, S. V. Morozov, D. Jiang, M. I. Katsnelson, I. V. Grigorieva, S. V. Dubonos, A. A. Firsov, Nature (London) 438, 197 (2005).

[27] P. D. Ye, D. Weiss, R. R. Gerhardts, M. Seeger, K. von Klitzing, K. Eberl, and H. Nickel, Phys. Rev. Lett. 74, 3013 (1995).

[28] S. V. Dubonos, A. K. Geim, K. S. Novoselov, J. G. S. Lok, J. C. Maan, and M. Henini, Physica E 6, 746 (2000).

[29] For a recent review see: A. Nogaret, J. Phys.: Condens. Matter 22, 253201 (2010).

[30] M. Nielsen and P. Hedegård, Phys. Rev. B 51, 7679 (1995). 
Graphene in inhomogeneous magnetic fields: Bound, quasi-bound, and scattering states30

[31] M. Zarenia, J. M. Pereira, F. M. Peeters, and G. A. Farias, Nano Lett. 9, 4088 (2009).

[32] M. Zarenia, J. M. Pereira, A. Chaves, F. M. Peeters, and G. A. Farias, Phys. Rev. B 81, 045431 (2010); ibid Phys. Rev. B 82, 119906(E) (2010). 